Portland State University

PDXScholar

$1-18-2022$

\title{
Random Lasing in Nano-Crystalline Zinc-Oxide Films
}

Benito Reynaldo Resendiz

Portland State University

Follow this and additional works at: https://pdxscholar.library.pdx.edu/open_access_etds

Part of the Materials Science and Engineering Commons, and the Nanoscience and Nanotechnology Commons

Let us know how access to this document benefits you.

\section{Recommended Citation}

Resendiz, Benito Reynaldo, "Random Lasing in Nano-Crystalline Zinc-Oxide Films" (2022). Dissertations and Theses. Paper 5894.

https://doi.org/10.15760/etd. 7765

This Thesis is brought to you for free and open access. It has been accepted for inclusion in Dissertations and Theses by an authorized administrator of PDXScholar. Please contact us if we can make this document more accessible: pdxscholar@pdx.edu. 


\title{
Random Lasing in Nano-Crystalline Zinc-Oxide Films
}

\author{
by
}

Benito Reynaldo Resendiz

A thesis submitted in partial fulfillment of the

requirements for the degree of

\section{Master of Science}

in

Physics

Thesis Committee:

Rolf Könenkamp, Chair

Andres La Rosa

Raj Solanki

Portland State University

2021 
(C) 2021 Benito Reynaldo Resendiz 


\begin{abstract}
In this thesis, we explore the preparation of random lasers (RLs) using solutiondeposited, randomly packed nano-particle films of zinc oxide $(\mathrm{ZnO})$ impregnated with silicon dioxide $\left(\mathrm{SiO}_{2}\right)$ nanospheres. RLs have their scatterers randomly oriented, while their lasing comes from light propagating along closed paths through the scattering environment. It is shown here that random lasing is readily observed in films made of submicron sized $\mathrm{ZnO}$ particles. Adding transparent $\mathrm{SiO}_{2}$ nanospheres to the films, we show there is an effective improvement of the lasing that is observable in all of the samples spectra. Specifically, we found that the lasing thresholds were lowered, the linewidths decreased, and the lasing output increased as compared to our pure $\mathrm{ZnO}$ films. In the best films the spheres occupied a volume of approximately $53 \%$. We also analyzed films with fluorescent pink poly Latex spheres (Latex spheres) in the $\mathrm{ZnO}$. These films did not produce random lasing, however, likely due to damage occurring in these polymeric spheres under optical pumping.
\end{abstract}




\section{Dedication}

This thesis is dedicated to my mother, Theresa Flores Resendiz, you've always taken care of me and have helped in every way you can; to my aunt and uncle, Rebecca Gloria Resendiz and Dr. Benito Rodriguez Resendiz: for inspiring me, nurturing my educational desires, and pushing me to be the best that I could be. Lastly, I dedicate this thesis to my family and friends who have supported me from the beginning of my academic career and throughout life. 


\section{Acknowledgements}

I would like to thank my advisor, Dr. Rolf Könenkamp, for his guidance and input during my research. I want to thank Dr. Andres La Rosa and Dr. Raj Solanki for being Committee members and helping me become a better physicist. I would also like to thank my peers Dr. Theodore Stenmark, Christopher Scheffler, and Nabila Islam for their advice throughout my academic tenor. Lastly, I would like to thank my professors and peers who have taught and challenged me every step of the way. 


\section{Table of Contents}

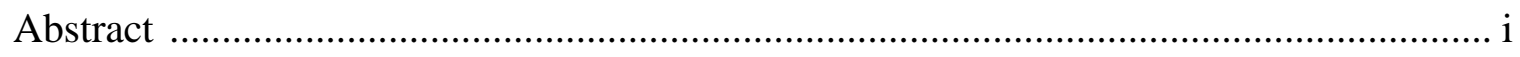

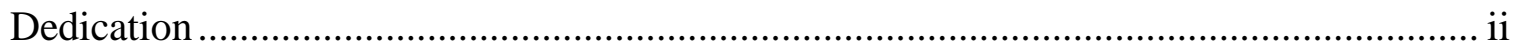

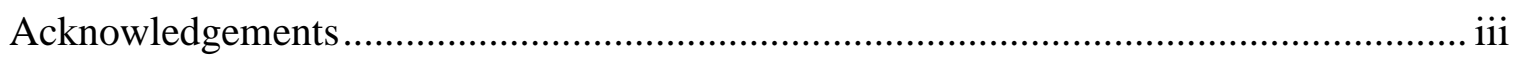

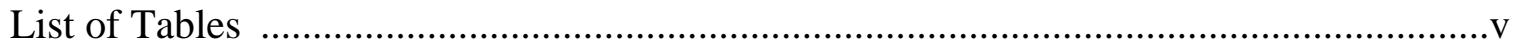

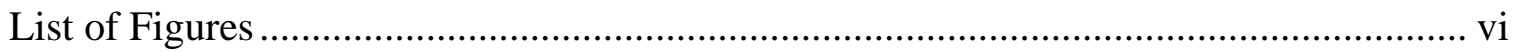

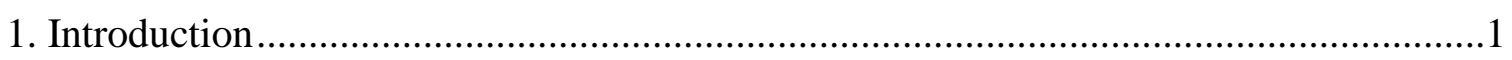

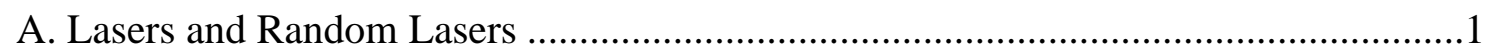

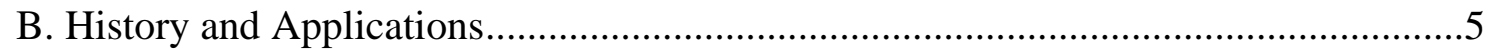

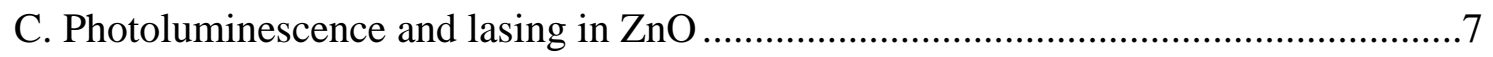

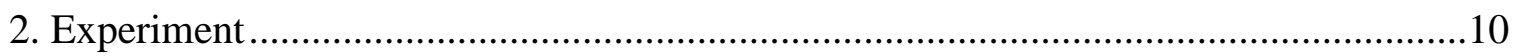

A. Material and Material Preparation ………………................................................10

B. Experimental Set Up …………………...................................................13

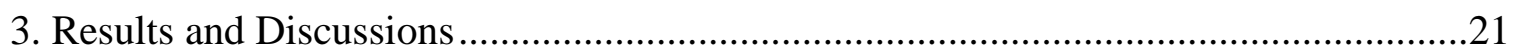

A. Operational limits at high pump laser intensities: Morphological changes in the $\mathrm{ZnO}$ film and at the Silicon mirror surface due to high pulse intensities..........................21

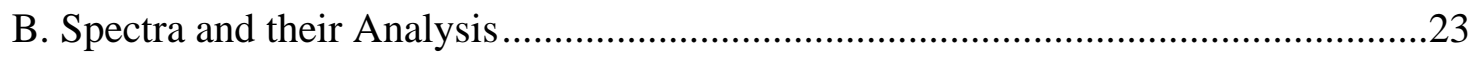

1. Spectra from $\mathrm{ZnO}$ Sample without Spheres........................................................23

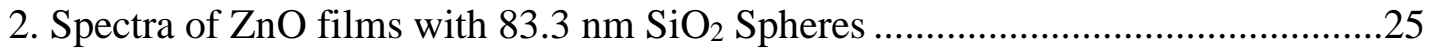

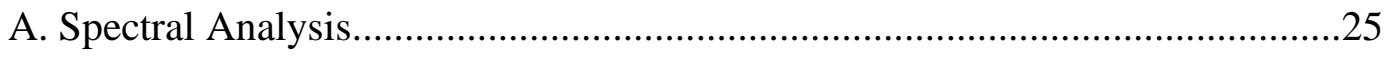

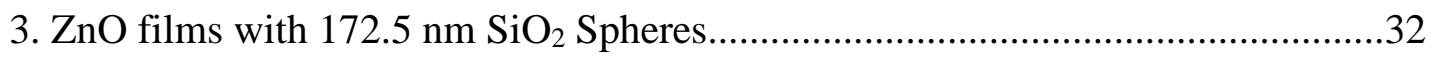

4. Summary for pure $\mathrm{ZnO}$ films and $\mathrm{ZnO}$ films with $\mathrm{SiO}_{2}$ Spheres ...........................35

5. Spectra from samples with Latex Spheres ............................................................38

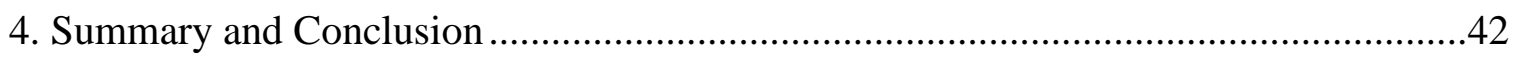

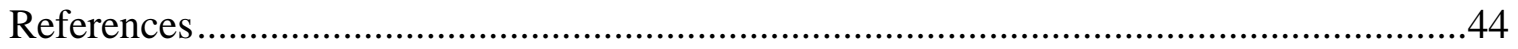




\section{List of Tables}

Table 1: The table displays the values of the lasing threshold, line narrowing threshold, smallest linewidth measured, and $R S V_{\text {Film }}$ 


\section{List of Figures}

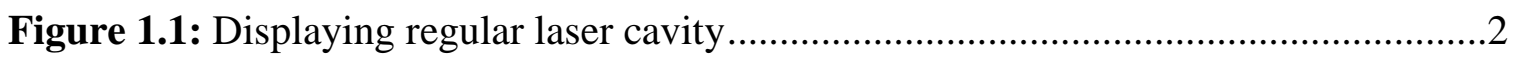

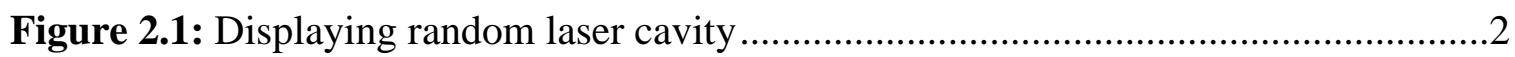

Figure 3.1: Demonstrates the path the laser travels with each of the parts labeled ..........14

Figure 4.1: Ray diagram of the laser spot being under focused, focused, and over focused

Figure 5.1: Measuring tape with $1 \mathrm{~mm}$ marks placed in orange .......................................17

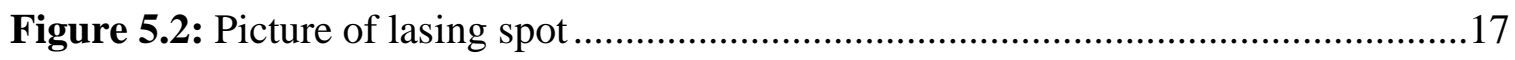

Figure 6.1: Concentration Factor vs. Lens Position X measured on the micrometer.......19

Figure 7.1: An area of thin film before laser exposure ..................................................22

Figure 7.2: The same portion of material after lasing damage had been observed with

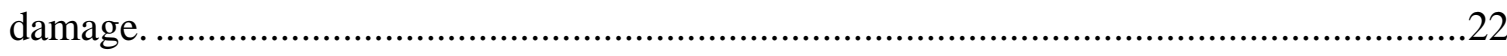

Figure 8.1: Spectra Plot for $\mathrm{ZnO}$ sample lasing after an increase in intensity with

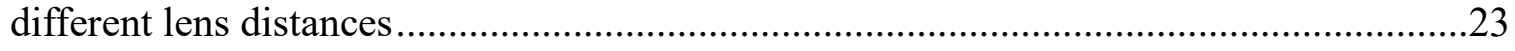

Figure 8.2: Normalization plot with different lens position ............................................23

Figure 8.3: Max Count vs Lens Position $X$ graph displaying lasing threshold ................23

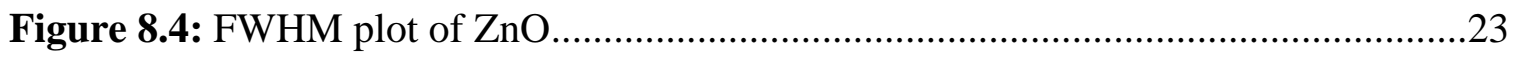

Figure 9.1: Spectra plot displaying a peak on the left-hand side as the intensity increases

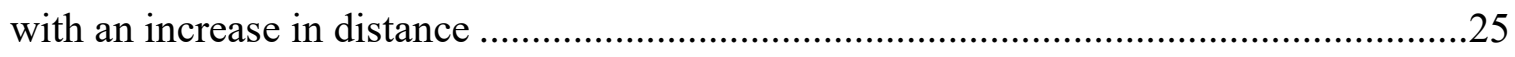

Figure 9.2: Normalized spectra plot for the collected distances .......................................25

Figure 9.3: Threshold plot of the Max Count vs. Lens Position X...................................25

Figure 9.4: FWHM data plot with lasing threshold labeled ...........................................25

Figure 10.1: Spectra plot displaying a peak on the left-hand side as the intensity increases with an increase in distance.........................................................................2

Figure 10.2: Normalized spectra displayed for the collected distances............................27

Figure 10.3: Plot of Max Count vs. Lens Position X....................................................27 
Figure 10.4: FWHM plot for the $0.4 \mathrm{ml} 83.3 \mathrm{~nm} \mathrm{SiO} 2$ sample

Figure 11.1: Spectra plot for $.8 \mathrm{ml} 83.3 \mathrm{~nm} \mathrm{SiO} 2$ sample lasing with increase in intensity with increase in distances.

Figure 11.2: Normalized spectra displayed for the collected distances

Figure 11.3: Displays the Max Count vs. Lens Position X graph with lasing threshold labeled.

Figure 11.4: FWHM with lasing threshold labeled .29

Figure 12.1: Spectra Plot of Emission Intensity vs. Wavelength. 31

Figure 12.2: Normalized graph of all the spectra plots .31

Figure 12.3: Threshold plot with Max Count vs. Lens Position X .31

Figure 12.4: FWHM plot with lasing threshold indicated 31

Figure 13.1: A spectra plot of Emission Intensity vs. Wavelength ...............................32

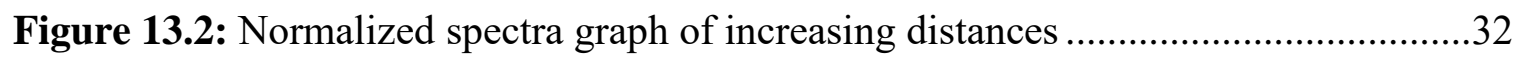

Figure 13.3: Threshold plot with Max Count vs. Lens Position X................................32

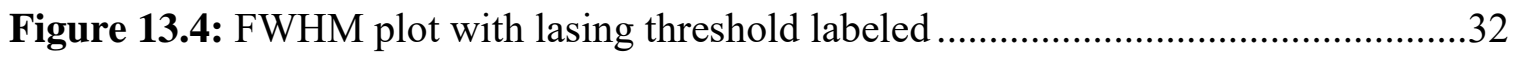

Figure 14.1: A spectra plot of Emission Intensity vs. Wavelength .................................34

Figure 14.2: Normalized spectra graph of increasing distances .................................34

Figure 14.3: A Max Count vs. Lens Position X displaying the lasing threshold..............34

Figure 14.4: FWHM plot with lasing threshold labeled ..........................................34

Figure 15.1: Threshold plot with Max Count vs. Lens Position X for all seven samples.

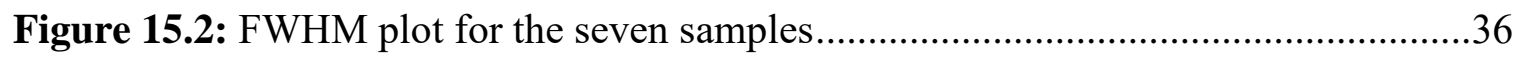

Figure 16.1: The graph displays the threshold $\mathrm{X}$ for intensity and line narrowing ..........37

Figure 17.1: Increasing spectra plot for the Latex sphere sample .................................38

Figure 17.2: Decreasing spectra plot for the Latex sphere sample ................................38 
Figure 17.3: Displays one spectrum plot to emphasize four peaks appearing on the sides of the gaussian peak that are located at $375.31,380.28,399.65$, and $405.78 \mathrm{~nm} \ldots \ldots \ldots \ldots . . .38$

Figure 17.4: The graph displays the change in Emission Intensity when the Pump

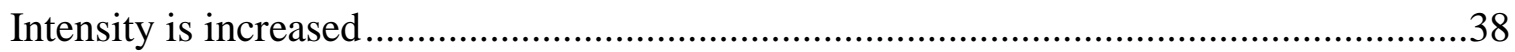

Figure 17.5: Linewidth plot for the Latex sphere sample for the narrow line at $405.78 \mathrm{~nm}$

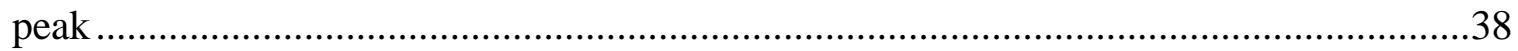

Figure 17.6: A table of known N2 lasing wavelengths ...........................................40

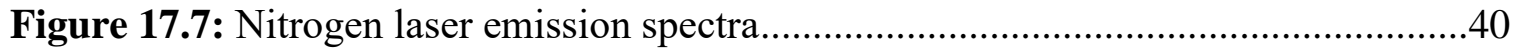




\section{Introduction \\ A: Lasers and Random Lasers}

When photons are absorbed by a semiconductor, they may give rise to electron transitions from the valence band to the conduction band. Conduction band states are then filled with electrons and the valence band has a reduced electron concentration. There is then an increasing probability for the incoming photons to generate a downward electron transition through de-excitation. A de-excitation of an electron from the conduction band can involve spontaneous light emission or stimulated emission.

Lasing can only be obtained when population inversion is reached; that is, when there is a higher occupation of electrons in high energy states than in lower energy states. Steady-state population inversion can usually not be obtained by optical pumping in a two-level system due to the rapid decay of the electron. Instead, a third or fourth energy level needs to be involved to obtain population inversion. For example, in a three-level system, electrons can decay from the top energy level to a level slightly below and if the decay from this lower level is slow, then an accumulation of electrons may occur in that level leading to population inversion [1]. The population inversion may further be improved with a four-level system.

When population inversion is reached through optical pumping, the incoming photons have a high probability of being stimulating photons. As the stimulating photons are not absorbed, there are then two photons for each incoming photon and the second photon generated has the same energy and momentum, polarization and phase as the stimulating photon. The newly generated photon may stimulate the emission of more 
photons and an avalanche of coherent
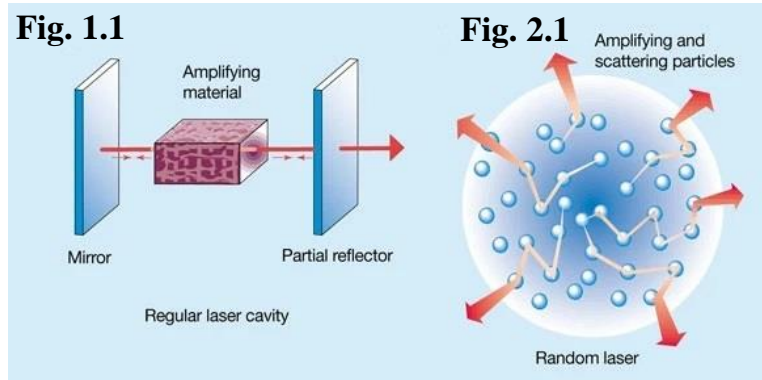

stimulated transitions constitute optical

gain. The lasing threshold is reached when

absorption and other optical losses are just

balanced by the optical gain. Above the

Figure 1.1: Displaying regular laser cavity.

Figure 2.1: Displaying random laser cavity.

- Figure 1.1 and 2.1 were taken from [4] Wiersma, Diederik. "The Smallest Random Laser." Laser Physics. vol. 406, num. 132133 (2000).

lasing threshold stimulated light emission prevails and "Light Amplification by

Stimulated Emission of Radiation" is obtained.

The lasing threshold is reached more easily when the lasing medium is located in an optical cavity. Such a cavity internally reflects the light a large number of times before it can leave the cavity volume. Therefore, the light intensity in the cavity is higher and the lasing threshold is reached more easily. In addition, the cavity may also change the spectral distribution of the light: Light that satisfies the resonance conditions of the cavity is enhanced, while off-resonance light is suppressed. The cavity acts as a resonator providing positive feedback for some wavelengths and negative feedback for others. A third important effect of the cavity is that it also defines the phase of the resonant radiation. Essentially, the resonant light in a cavity has a fixed phase and that includes the stimulated light. This means that the cavity may increase the degree of coherence of the laser light. Typically, only a small portion of the light is allowed to escape from the cavity through a window of reduced reflectivity. 
Strong stimulated emission is unachievable in non-inversion or in thermal equilibrium conditions. In these cases, there is always a larger probability for a photon to generate an upward transition of an electron through absorption. As the photon is consumed in the upward transition, there is no gain. Gain can only be obtained when there is a larger probability for a downward transition [3], i.e. for the case of population inversion. The lasing efficiency, the spectral properties and the coherence of a laser depend on the feedback provided by the resonator. A laser of this type is displayed in Fig. 1.1 .

Population inversion may be facilitated and modified by feedback. In conventional lasers, feedback is obtained from an optical cavity and the cavity properties largely determine spectral linewidth, coherence, and efficiency. In a random laser (RL) there are no designed cavities but scattering typically occurs at randomly oriented grain boundaries in a solid material or other random inhomogeneities in solids or liquids. The light intensities achieved in this random scattering are usually insufficient to reach the lasing threshold; therefore, high intensity pulsed laser light is often used for pumping RLs. For generating significant coherence and obtaining narrow linewidths some type of feedback mechanism is needed. In a random environment, a minimum of feedback could be provided by a single closed optical path that brings back some of the photons to the area where they were created.

Once a closed path is established, a feedback loop is obtained, coherence for stimulated photons increases, and resonant line narrowing may occur. However, the 
degree of coherence and the strength of any resonance depends on the details of the closed optical path; in particular, how much it contributes to the total intensity of the light. If its contribution is small, the degree of coherence will be small; therefore, the effect on linewidth and coherence will be small. In that case one may use the term nonresonant feedback RL. If the closed optical loop establishes strong resonant feedback, one may expect higher coherence, and sharper spectral lines. The RL is displayed in Fig. 2.

There are key differences between traditional lasers and RLs relating to the principals of how they work, their construction, and their optical properties. Both types of lasers need population inversion to achieve lasing. Traditional lasers use an optical resonator to recycle the pump light, thereby increasing efficiency, mode selection, higher coherence, and narrower spectral linewidths of the emitted light. RLs do not have a specifically designed optical cavity, but random scattering at grain boundaries may lead to closed optical paths; yet often, with low probability [4]. Accordingly, RLs typically have lower efficiency, lower coherence, and broader linewidth than traditional lasers. The lack of symmetry in the optical closed path may also limit the directional character of the lasing light. While designed resonators typically give high directionality, RLs tend to have very low directionality.

The near parallelity of rays in traditional lasers correlates with their divergence being very small. RLs instead have a large divergence [5]. Traditional lasers can be made in multiple ways, such as a gas laser, dye laser, ion laser, solid-state laser or electrically pumped diode lasers. RLs are limited to the multi-crystalline solid-state form or consist 
of powders, particle suspensions or dye molecular solutions, as they need grain boundaries or other inhomogeneities to function as scatterers.

\section{B. History and Applications}

While Einstein predicted the principle of lasing in early theoretical work [6], there has been dispute as to a single inventor of the first laser device. There now seems to be a general consensus that there is no single inventor. The most fundamental contributions made in the U.S. were done by Charles Townes, Leonard Schawlow, and Nicolaas Bloembergen. The discovery of non-resonance energy feedback in RLs was attributed to the pioneering work of V. S. Letokhov and co-workers during the 1960s [7].

The preparation of ordinary laser crystal often involves rather complex crystal growth methods that can easily exceed $700{ }^{\circ} \mathrm{C}$ while RL preparation is typically much simpler and involves comparably lower temperatures. RL action has been observed in simple semiconductor powders, such as $\mathrm{ZnO}$ or gallium nitride $(\mathrm{GaN})$, nanostructured and non-nanostructured thin films, ceramics, and laser dye solutions. Hence, the production cost for RLs can be much lower while traditional lasers often require more expensive materials.

There has been research into various applications of RLs in topics that involve a nematic liquid crystal (LC) that has a high refractive index and a variety of optical functionalities. The LC research involved dye-doped chiral nematic LCs that spontaneously form a distributed laser with a spatially periodic helical structure. In other work it was also demonstrated that whispering gallery modes (WGMs) could be realized 
by using the dye-doped LCs due to the cavities found within the material and the gain medium. There are applications with polymer-dispersed LCs, and amplification of light within random lasing (RLg) [8].

RLg has been achieved within a granular gaseous system with the use of sound vibrations from a subwoofer and optical stimulation from a laser. The purpose of this experiment was to demonstrate that RLg could be achieved through mechanical and optical means. The sample was a liquid fluorescent solution of rhodamine B with 1,500 nonmagnetic steel spherical metallic grains with a diameter of 1 millimeter $(\mathrm{mm})$. The addition of the metallic spheres was to increase the probability of cavities being formed from RLg. The solution was placed on top of a vertically driven vibrating plate that oscillated at an amplitude produced by the subwoofer. The RLg signal arose once the subwoofer vibrated the plate to an oscillation of $70 \mathrm{Hertz}(\mathrm{Hz})$ and this is when the grains would have a granular gaseous state. The sample was also pumped with a laser beam of $532 \mathrm{~nm}$ at $7 \mathrm{~ns}$ pulse durations causing the sample to emit a RLg peak at $600 \mathrm{~nm}$. When vibrations reached a slightly greater frequency than $70 \mathrm{~Hz}$, another peak was emitted at $620 \mathrm{~nm}[9]$.

A research team from the National Taiwan University made 3 random monochromatic polymer film organic laser dyes that demonstrated red $(620 \mathrm{~nm})$, green $(562 \mathrm{~nm})$, or blue $(465 \mathrm{~nm})$ lasing signals. The samples were highly pure and stable with wavelength, line width, and intensity that were nearly isotropic. The green sample had silver nanoparticles to help as plasmonic scattering centers that had a radius of $10 \mathrm{~nm}$. The team demonstrated how the emission spectra looked individually then with different 
combinations of the three substrates, showed the laser spectra at various different angles $\left(25,45,65,85^{\circ}\right)$ and recorded white lasing values [10].

In frequency ranges where high-reflectivity mirrors are not available (e.g., gamma-rays, x-rays), the feedback provided by an appropriate scattering medium can be used as an alternative to generate laser action. There has been some demonstration within photonic barcode, opto-microfluidics, optical batteries, cancer diagnostic, speckle-free bio-imaging, on-chip random spectrometer, and time resolved microscopy/spectroscopy.

\section{Photoluminescence and lasing in $\mathrm{ZnO}$}

$\mathrm{ZnO}$ is a semiconductor with an energy bandgap of 3.34 electron Volts $(\mathrm{eV})$ at room temperature [11]. Thus, the excitation of a valence band electron to the conduction band requires a photon of minimum energy of $3.34 \mathrm{eV}$ or a wavelength of $371.2 \mathrm{~nm}$. Photoluminescence (PL) is obtained when a photon excites an electron from the valence band to the conduction band and the excited electrons and holes subsequently recombine and send out photons. However, the photon energy in the recombination process is usually not equal to the photon energy of the excitation process but is more similar to the bandgap energy.

Indeed, in $\mathrm{ZnO}$ the PL energy at room temperature is found to be $3.25 \mathrm{eV}$ corresponding to a wavelength of $384 \mathrm{~nm}$ and this value is fairly independent of the excitation energy. The $3.25 \mathrm{eV}$ in PL are seen to be slightly smaller than the bandgap energy of $3.34 \mathrm{eV}$. This indicates that the electron transitions in PL are not exclusively from the conduction band edge to the valence band edge but may involve other energy states. In $\mathrm{ZnO}$ the PL involves crystal defects, excitons, and phonons [12]. 
An exciton is a bound state of an electron and a hole which are attracted by the electrostatic Coulomb force [13]. This attraction provides a stabilizing energy balance for the electron-hole pair. The exciton is electrically neutral and exists in insulators, semiconductors, and in some liquids. The exciton can transport energy without transporting net electric charge and can form when a photon is absorbed by a semiconductor. The exciton has slightly less energy than the unbound electron and hole. The exciton level is energetically below the bandgap energy and this facilitates population inversion and makes the material a candidate for UV lasing. Lasing in $\mathrm{ZnO}$ has been observed by Sun et al. at a wavelength of $388 \mathrm{~nm}$ and this wavelength has been attributed to recombination in an electron-hole plasma [14].

In our experiments with $\mathrm{ZnO}$ nanocrystals the lasing is also observed at a wavelength of $\sim 388 \mathrm{~nm}$, while the PL peak is typically seen between $385 \mathrm{~nm}$ and $395 \mathrm{~nm}$ with a linewidth of $\sim 10-13 \mathrm{~nm}$. These small variations may be due to the lower crystalline quality of the nanomaterial. Lasing radiation typically has a much narrower spectral linewidth than PL. In our case of RLg, line widths as small as $2 \mathrm{~nm}$ are observed.

Random lasing in $\mathrm{ZnO}$ [15] has been well studied in the past few years with particular emphasis on $\mathrm{ZnO}$ films with added materials or processes to enhance the lasing. Pure $\mathrm{ZnO}$ films made of powders with varying grain sizes and different grain connectivities have been studied [16]. The effect of different film morphologies has also been studied with comparisons between poly-crystalline films, films of near-vertical nano-sheets [17] and nano-needle films [18, 19]. Also, discrete particle films can be mentioned here, which allow the possibility of obtaining very narrow single lasing lines 
[20]. Optical enhancement in the $\mathrm{ZnO}$ films has been studied by interspersing polymers [21], plasmonic metal particles [22, 23], and by adding $\mathrm{ZnO}$ to dye solutions [24]. Also, films with spherical $\mathrm{ZnO}$ particles have been prepared and studied [25, 26]. Many of these alterations going beyond uniform $\mathrm{ZnO}$ films have shown improvements in the random lasing properties, i.e. lowering of the lasing threshold values, narrowing of the lasing line width and stronger gain.

In this thesis we explore the possibility of using transparent spherical silica and Latex spheres of subwavelength diameter in loosely-packed $\mathrm{ZnO}$ films to improve the RLg [27]. With the introduction of these scattering elements, the light propagation in the $\mathrm{ZnO}$ powder films is modified and we observe an improvement of the quality and strength of the lasing.

We characterize the lasing by its threshold behavior and by the line narrowing taking place at the lasing transition. 


\section{Experiment}

\section{A. Material and Material Preparation}

The $\mathrm{ZnO}$ nanoparticles that were used here had average particle sizes of around 240 $\mathrm{nm}$ and were purchased from MKNano Inc. The $\mathrm{SiO}_{2}$ nanospheres were purchased from Corpuscular Inc. with average diameter sizes of 83.3 and $172.5 \mathrm{~nm}$. The spheres were delivered as suspensions in ethanol with a sphere volume concentration of 5\%. The Latex spheres with a mean diameter of $.86 \mu \mathrm{m}$ were purchased from Spherotech Inc. These spheres also came as suspensions in ethanol with a sphere volume concentration of 5\%. The purpose of having premade spheres was to save on time, resources, and to have consistency with their sizes. The index of refractions for the materials are as follows: air is $1, \mathrm{SiO}_{2}$ is 1.5 , Latex is 1.59 , and $\mathrm{ZnO}$ is between 1.6 and $2-$ depending on preparation.

$\mathrm{ZnO}$ has two main crystalline forms, hexagonal wurtzite and cubic zincblende [28]. We are working with the wurtzite structure. Wurtzite structure is the most common and stable in ambient conditions. $\mathrm{SiO}_{2}$ has many different crystalline forms, which are referenced as polymorphs. We are working with $\mathrm{SiO}_{2} \alpha$-quartz. The $\mathrm{SiO}_{2} \alpha$-quartz will not morph into the next form, $\beta$-quartz, until $573{ }^{\circ} \mathrm{C}$. In our preparation process the highest temperature experienced by the spheres was during the drying of the sphere solution on the substrate which was $100{ }^{\circ} \mathrm{C}$. There is also no worry about the spheres being damaged or melting during the annealing or the optical pumping process because the melting point of $\mathrm{SiO}_{2}$ is $1,600{ }^{\circ} \mathrm{C}$ [29]. The spheres themselves are transparent, passive dielectrics without gain. These spheres have absorption coefficient and index of refraction smaller than the loosely-packed $\mathrm{ZnO}$ and may extend the scattering length for 
light in the films. The Latex spheres are a form of polystyrene and contain dye molecules that make them fluorescent. The melting point of polystyrene is $240{ }^{\circ} \mathrm{C}$ [30].

The nanoparticle films were prepared on a quartz substrate using particle suspensions of 5 milliliters $(\mathrm{ml})$ of $95 \%$ ethanol/ $\mathrm{H}_{2} \mathrm{O}$. The thin film preparation used a combination of dip coating and drop coating processes with suspensions of the nanospheres and $\mathrm{ZnO}$ nanoparticles.

For the pure $\mathrm{ZnO}$ films, $.5 \mathrm{~g}$ of $\mathrm{ZnO}$ was weighed out and annealed at $550{ }^{\circ} \mathrm{C}$ for one hour. The $\mathrm{ZnO}$ was then placed into a beaker with $10 \mathrm{ml}$ of ethanol and the solution was sonicated for ten minutes. The quartz slide was dipped in the $\mathrm{ZnO}$ solution producing 10 layers and after each layer deposition the slide was dried on a hot plate in air at $100{ }^{\circ} \mathrm{C}$.

For the samples with $\mathrm{SiO}_{2}$ spheres we made a total of six samples. Four samples with $83.3 \mathrm{~nm} \mathrm{SiO} 2$ spheres were prepared in the following way: .2 $\mathrm{g}$ of $\mathrm{ZnO}$ powder was annealed for one hour at $550{ }^{\circ} \mathrm{C}$ and was then added to $10 \mathrm{ml}$ ethanol and this solution was then sonicated for 10 minutes. Varying amounts of $83.3 \mathrm{~nm} \mathrm{SiO} 2$ sphere solutions (.2 $\mathrm{ml}, .4 \mathrm{ml}, .8 \mathrm{ml}$, and $2 \mathrm{ml}$ ) were then added to the $\mathrm{ZnO} /$ ethanol solution to obtain 4 sample solutions. These were then sonicated for another 10 minutes. Nano-particle films were then deposited onto a quartz from the four solutions in layer form, each with a total of ten dipped layers and three micro-pipetted layers with each layer dried on a hot plate at $100{ }^{\circ} \mathrm{C}$. Each micro-pipetted layer used $.018 \mathrm{ml}$ of nano-particle/sphere solution.

Two samples with spheres of $172.5 \mathrm{~nm}$ diameter were made with $.4 \mathrm{ml}$ of sphere solution and the preparation process was the same for both samples: $.2 \mathrm{~g}$ of $\mathrm{ZnO}$ powder 
were annealed at $550{ }^{\circ} \mathrm{C}$ for one hour. The powder was then placed into a beaker of $10 \mathrm{ml}$ of ethanol and sonicated for 10 minutes. $.4 \mathrm{ml}$ of the $\mathrm{SiO}_{2}$ sphere solution was then added and the combined solution was sonicated for another 10 minutes. The first sample used a volume of $.01 \mathrm{ml}$ of the $\mathrm{SiO}_{2} / \mathrm{ZnO}$ solution for one micro-pipetted layer that was dried at $100{ }^{\circ} \mathrm{C}$ on a quartz substrate. The second sample had three micro-pipetted layers with each layer using $.01 \mathrm{ml}$ of the $\mathrm{SiO}_{2} / \mathrm{ZnO}$ solution and each layer was annealed at $100{ }^{\circ} \mathrm{C}$.

For these films the relative sphere volume $R S V_{\text {Film }}$ was calculated as follows:

$$
R S V_{\text {Film }}=\frac{V S_{\text {Solution }}}{V S_{\text {Solution }}+V Z_{\text {Solution }}} * 100 \%
$$

where $R S V_{\text {Film }}$ means the relative sphere volume in the film, $V S_{\text {Solution }}$ means the volume of spheres in the sphere solution and $V Z_{\text {Solution }}$ means the volume of $\mathrm{ZnO}$ nanoparticles in the $\mathrm{ZnO}$ solution. $V S_{\text {Solution }}$ and $V Z_{\text {Solution }}$ are each determined by multiplying the solution concentrations with the solution volume. We used sphere solution volumes of $.2 \mathrm{ml}, .4 \mathrm{ml}, .8 \mathrm{ml}$, and $2 \mathrm{ml}$ and kept the $\mathrm{ZnO}$ solution volume constant at $10 \mathrm{ml}$. The resulting $R S V_{\text {Film }}$ in the films were as follows:

For the $.2 \mathrm{ml} 83.3 \mathrm{~nm} \mathrm{SiO}_{2}$ sphere sample the relative sphere volume was $22 \%$, for the $.4 \mathrm{ml} 83.3 \mathrm{~nm} \mathrm{SiO} 2$ sphere sample the relative sphere volume was $36 \%$, for the $.8 \mathrm{ml} 83.3 \mathrm{~nm} \mathrm{SiO} 2$ sphere sample the relative sphere volume was $53 \%$, for the $2 \mathrm{ml} 83.3 \mathrm{~nm} \mathrm{SiO}_{2}$ sphere sample the relative sphere volume was $73 \%$, for the two $.4 \mathrm{ml} 172.5 \mathrm{~nm} \mathrm{SiO}_{2}$ sphere samples the relative sphere volume was $36 \%$.

For the samples with Latex spheres, the procedure consisted of dip coating and drop coating sequences. Latex sphere solutions with a volume concentration of $5 \%$ were 
micro-pipetted at two different stages of the $\mathrm{ZnO}$ layer preparation. .2 $\mathrm{g}$ of $\mathrm{ZnO}$ powder was annealed at $550{ }^{\circ} \mathrm{C}$ for one hour. The powder was then placed into a beaker of $10 \mathrm{ml}$ of ethanol and sonicated for ten minutes. First, 5 layers of $\mathrm{ZnO}$ were dip coated onto the substrate with the latex sphere layer micro-pipetted onto the surface after the $5^{\text {th }} \mathrm{ZnO}$ layer. Next, 5 more layers of $\mathrm{ZnO}$ were dip coated onto the substrate with the second Latex sphere layer micro-pipetted after the $10^{\text {th }} \mathrm{ZnO}$ layer. Finally, 4 more $\mathrm{ZnO}$ layers were added to the sample using dip-coating. The solution volumes of the Latex spheres were .003 and $.001 \mathrm{ml}$ respectively.

\section{B. Experimental Set Up}

The experimental set up is shown in Fig. 3.1. The experiment uses a PL 2300 Nitrogen UV Pulse Laser, made by Photon Technology International, and the laser has a wavelength of $337 \mathrm{~nm}$. An AmScope Camera connected to the microscope producing a live video feed. An optical cable was connected to an Ocean Optics spectrometer with .7 $\mathrm{nm}$ wavelength resolution and an Ocean Optics SpectraSuite program that was used to interpret the collected data. The laser produces a pulse that has a duration of 800 picoseconds (ps) and a maximum pulse energy of 2.8 millijoules $(\mathrm{mJ})$ [31]. In earlier work, it was found that working with pico-second pulses produce spectra that are reproducible with reliable, consistent data for the spectrum. Exposing the lasing samples to longer timed pulses lacked reproducibility. 
The UV laser pulses travel through a shutter, an aperture, and are then concentrated by a lens. The pulse is reflected off a silicon $(\mathrm{Si})$ wafer to illuminate the

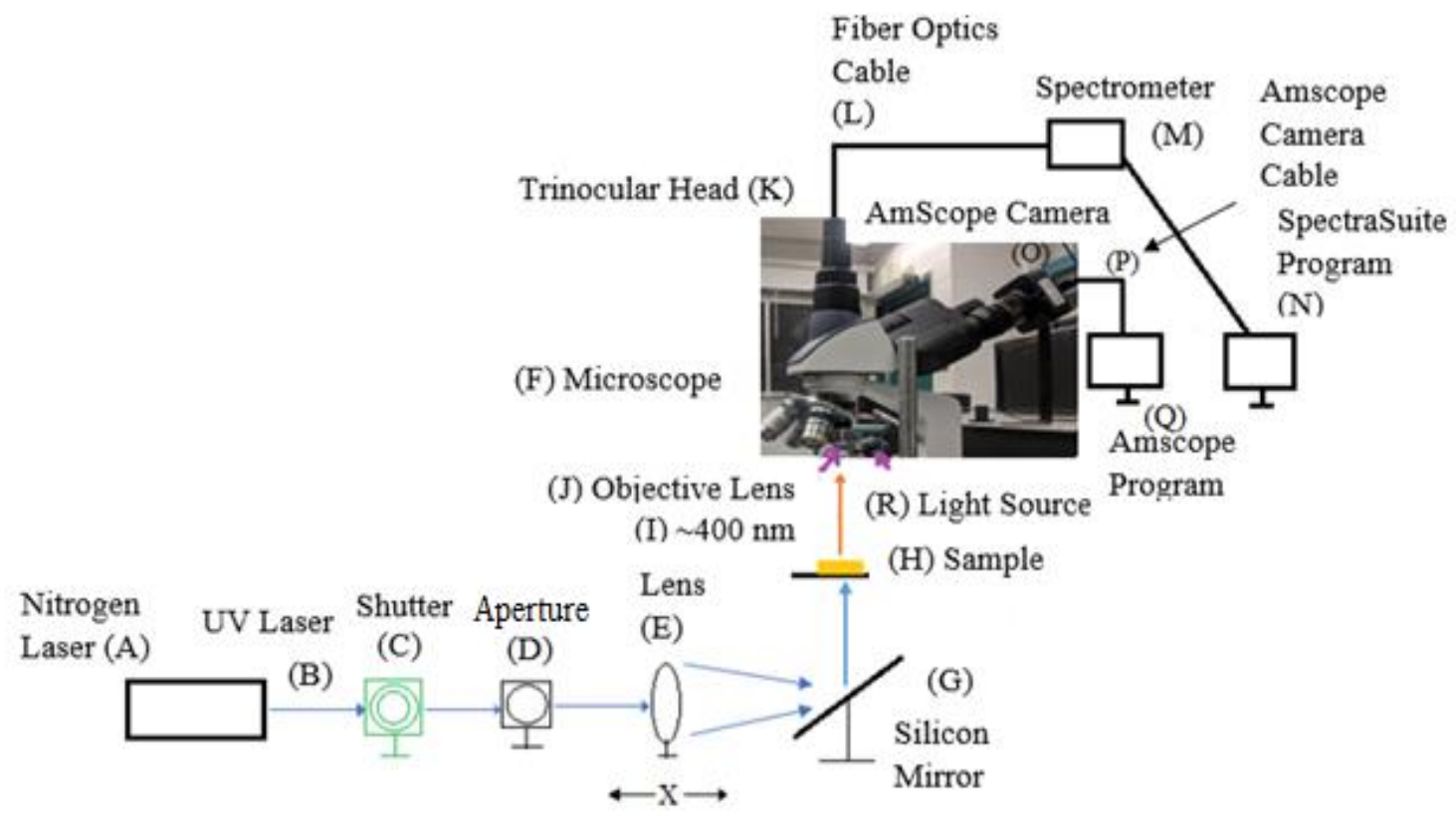

Fig. 3.1: Demonstrates the path the laser travels with each of the parts labeled.

$\mathrm{ZnO} /$ nanosphere film from below, i.e. through the quartz substrate. The light signal emitted from the $\mathrm{ZnO} /$ nanosphere film will travel upward through the UV objective lens of the microscope and into the trifocal microscope top. A fiber optics cable connects the microscope to the spectrometer and camera. The spectrometer is read out using an OceanOptics SpectraSuite program that will display the light emitted from the sample as a spectrum with intensity counts as a function of wavelength [32].

The intensity of the nitrogen laser pulse on the sample was adjusted with a lens that had been placed on a $\mathrm{x}-\mathrm{y}$ stage with a micrometer reading in the $\mathrm{x}$-direction. We define the $\mathrm{x}$-direction to be in the beam direction of the pump laser. For taking a series of spectra, the nitrogen laser intensity at the location of the sample is regulated by focusing 
the lens. As the laser spot becomes more focused, there is an increase in intensity on the sample, while the total number of photons illuminating the spot remains constant.

We also visually observed the spot where the laser was focused. The AmScope digital camera was mounted in the left microscope eye piece, providing a live video feed, and enabling us to take images of the sample in real time with a magnification of 10x. The lens could be moved in the $\mathrm{x}$-direction by a total distance of $30 \mathrm{~mm}$ with the vast majority of our collected data coming from between $0.0 \mathrm{~mm}$ to $25 \mathrm{~mm}$.

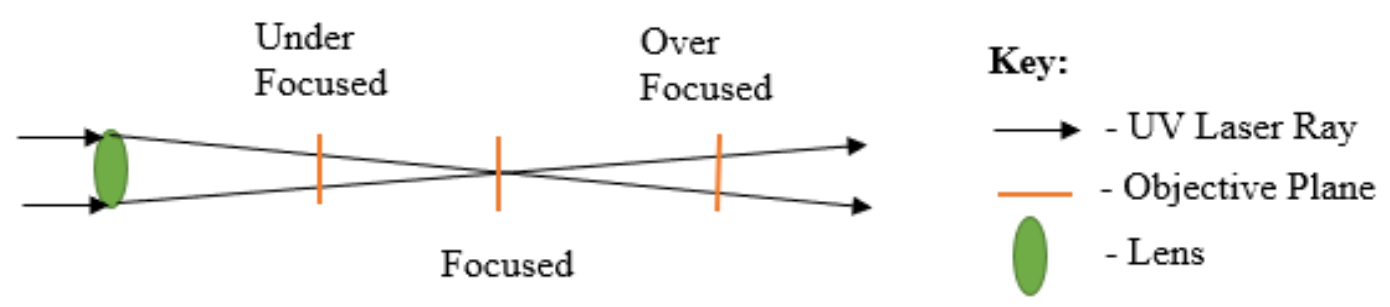

Fig. 4.1: Ray diagram of the laser spot being under focused, focused, and over focused.

The lens focal length was $80 \mathrm{~mm}$. The UV pump lasing radiation from the N2laser is absorbed in the $\mathrm{ZnO} /$ nanosphere film producing $\mathrm{PL}$ and $\mathrm{RLg}$. The emitted light signal from the sample typically covers a wavelength spectrum in the range $370 \mathrm{~nm}$ to $420 \mathrm{~nm}$.

The ray diagram in Figure 4.1 is modeling the pump laser ray path and the lens focal point on the sample surface. When the lens is very-far away from the sample surface, the laser beam is overfocused, and the exposure spot intensity is low. As the lens is moved closer to the sample, the focal point approaches the sample, the spot size is 
decreased, and the spot intensity increased. Once the lens focal point moves beyond the sample, the laser beam becomes under focused, and the spot intensity drops again as the lens moves further away.

The way that the illuminated area was estimated was that there was a picture taken of the laser spot with a piece of paper on the sample holder, then converted from pixels to $\mathrm{mm}$ using a piece of measuring tape that was placed on the sample holder as displayed in Figure 5.1. $1 \mathrm{~mm}$ was estimated to be equal to 270 pixels which was the difference between 490 pixels and 220 pixels. The 220 and 490 pixel are measuring points that are emphasized in Figure 6.1 with orange lines. The area estimated for the lasing spot is in Figure 5.2. The formula for calculating the area of an oval for the laser spot is: Area $=$ width $*$ height $* \pi$. The area was calculated to be $.7222 \mathrm{~mm} * .4629 \mathrm{~mm}$ $* \pi=1.05025446 \mathrm{~mm}^{2}$. The values obtained for the width and height were obtained from point differences. The width points were 275 and 470 pixels with their difference being 195 pixels. This was divided by 270 pixels to get $.72 \mathrm{~mm}$. The two measuring points for height were 15 and 140 pixels with the difference being 125 pixels. 125 pixels was divided by 270 pixels to equal $.4629 \mathrm{~mm}$. 


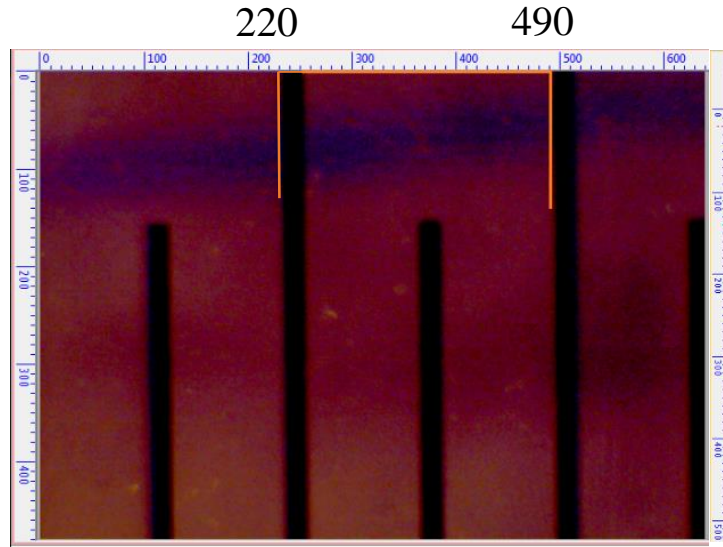

Figure 5.1: Measuring tape with $1 \mathrm{~mm}$ marks placed in orange.

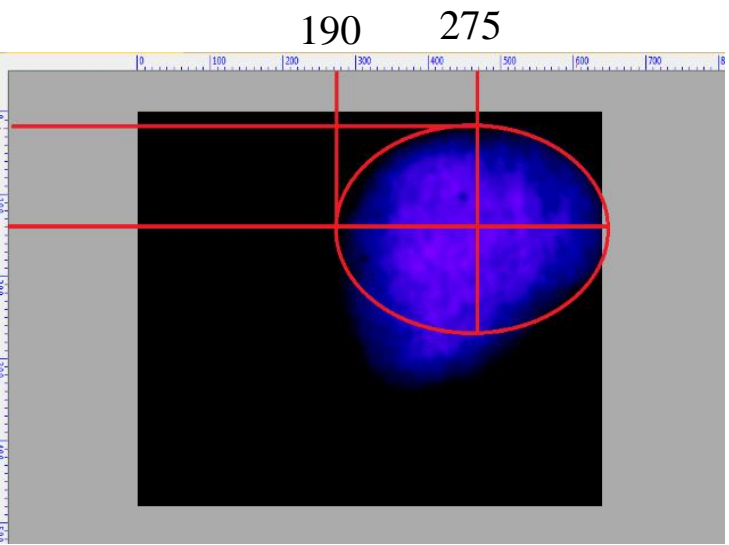

Figure 5.2: Picture of lasing spot.

The pump laser intensity, i.e. light intensity delivered by the nitrogen pump laser in each pulse is:

$$
\left(I_{o}\right)=\frac{\operatorname{Power}(P)}{\operatorname{Area}(A)}=\frac{1.45 \mathrm{~mJ} / 800 \mathrm{ps}}{B * C * \pi}=\frac{1.81 * 10^{6} \mathrm{~J} / \mathrm{s}}{1.05025 \mathrm{~mm}^{2}}=1.73 * 10^{6} \mathrm{~W} / \mathrm{mm}^{2}
$$

Behind the focusing lens this intensity changes at every point. The pump intensity at the sample surface is calculated with the formula:

$$
I_{\text {sample }}=I_{O} * F
$$

Where $I_{\text {sample }}$ is the intensity of the laser spot on the sample surface and $F$ is the concentration factor obtained by the focusing of the lens.

We used equation (eq.) (3) to obtain an estimate for the concentration factor $F$ :

$$
F=\frac{1}{\left(1-X_{1} / L_{F}\right)^{2}+A_{F} / A_{L}}
$$


When $A_{L}$ is the spot size of the un-collimated laser beam, $A_{F}$ is the smallest achievable spot size, $X_{1}$ is the distance of the lens from the film surface and $L_{F}$ is the focal length of the lens, which is $80 \mathrm{~mm}$. Hence, when $X_{1}=L_{F}$, the lens is focused on the sample surface. At this point aberrations and diffraction put a limit on the spot size in the focal point of the lens. We estimate the smallest achievable spot size for our set up to be $A_{F}=.022 \mathrm{~mm}^{2}$ based on measurements and microscope images. The un-collimated spot size-was 1.05 $\mathrm{mm}^{2}$.

In the experiment we read the position of the lens on the micrometer which is offset from $X_{1}$ by a distance of $50 \mathrm{~mm}$. In other words, we have $X_{1}=\mathrm{X}+50 \mathrm{~mm}$, where $\mathrm{X}$ is the micrometer reading. Inserting this relation into eq. (2) we can express the concentration factor $F$ directly in terms of the micrometer reading $X$ as

$$
F=\frac{1}{(1-(X+50 \mathrm{~mm}) / 80 \mathrm{~mm})^{2}+0.022}
$$

This function is plotted in Fig. 6.1. Typical micrometer readings in the experiments are in the range $0<X<25 \mathrm{~mm}$ resulting in concentrations factors $F$ in the range from 7 to 45 . Damage typically occurred in the samples for $X>27 \mathrm{~mm}$. 


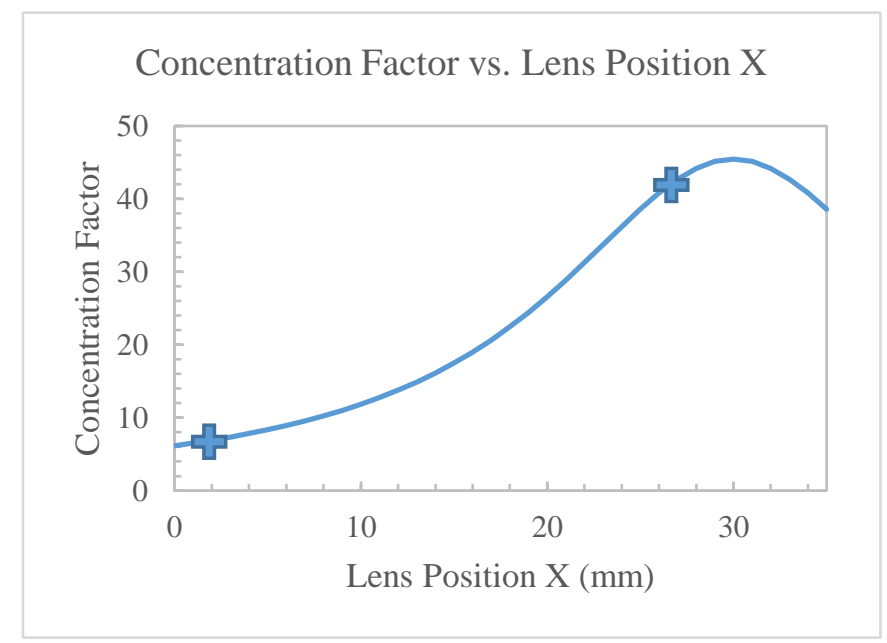

Figure 6.1: Concentration Factor vs. Lens Position X measured on the micrometer.

What we see in Fig. 6.1 is the intensity increase as the position $\mathrm{X}$ of the lens is moved towards the sample. The maximum concentration is reached when the lens position on the micrometer reading is at $30 \mathrm{~mm}$. Two experimental data points (shown as blue crosses) for this curve were calculated from figures 5.2 and 7.2.

When the lens is moved, the intensity at the sample surface changes while the total photon number remains constant. Accordingly, as long as the sample only produces PL, the light output from the sample is expected to remain constant. When the lens is moved, however, the lasing threshold is reached and lasing begins, and the sample light emission is expected to increase rapidly. At the same time, a smaller linewidth is expected to occur in the emitted light, as the laser linewidth is typically smaller than the PL linewidth. A steep intensity change and a spectral line narrowing are thus two characteristic properties of the sample emission that we can observe. Our spectrometer 
has a wavelength resolution of $.7 \mathrm{~nm}$ such that we can expect to resolve linewidths $>1$ $\mathrm{nm}$. We can thus evaluate the transition to lasing in two different plots: First, in a threshold plot showing sample light emission intensity vs. pump intensity. Second, in a plot showing the sample spectral linewidth vs. pump intensity. We will use these plots in the experimental evaluation. 


\section{Results and Discussions}

In this section we will present the results of our optical experiments performed on a variety of sample structures. We start out with a discussion of the operational limits set by laser damage occurring in the $\mathrm{Si}$ mirror and in the $\mathrm{ZnO} /$ nanosphere films themselves. We then present results showing PL and RLg in pure $\mathrm{ZnO}$ films, followed by results on $\mathrm{ZnO}$ films containing various types of silica nanospheres. These results will focus on the observation of a lasing threshold and on spectral linewidth narrowing as indicators for RLg. At the end of this section, we will discuss results obtained on the $\mathrm{ZnO}$ film containing latex spheres. These are the only films where RLg was not observed.

\section{A. Operational limits at high pump laser intensities: Morphological changes in the $\mathrm{ZnO}$ film and at the Silicon mirror surface due to high pulse intensities}

There is some morphological damage that the samples experience at high pump intensities [33] and we were able to observe surface damage on the films induced by the laser in real time. An example of such laser damage is shown in Figs. 7.1 and 7.2. At too high of an intensity, we saw the $\mathrm{ZnO}$ sample get burned clean off of the quartz slide. This typically occurred at lens positions where $X>27 \mathrm{~mm}$. Through trial and error, we were able to find lens distances that allowed for the study of the sample without inflicting damage. We were also able to conclude that the samples would continue to show lasing, even if sections had become porous or had broken apart. In these cases, the pump laser would shine through the sample but as the pump laser wavelength was not transmitted through microscope, the $\mathrm{ZnO}$ light emission spectra could still clearly be recorded. On the other hand, a too thick and solid $\mathrm{ZnO}$ film typically showed reduced $\mathrm{ZnO}$ light 
emission, yet threshold and line narrowing could, in most cases, still be observed.

Over time, the spot where the laser spot was reflected off of the Si mirror would get damaged or eroded causing distorted and reduced pump intensity on the sample. In these cases, we moved the Si mirror so that an area that was damaged would not reflect the pump laser light.

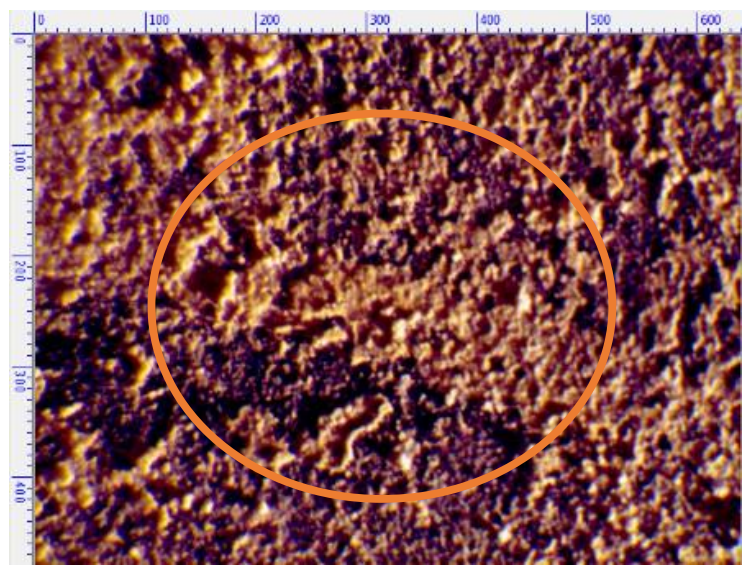

Figure 7.1: An area of thin film before laser exposure.

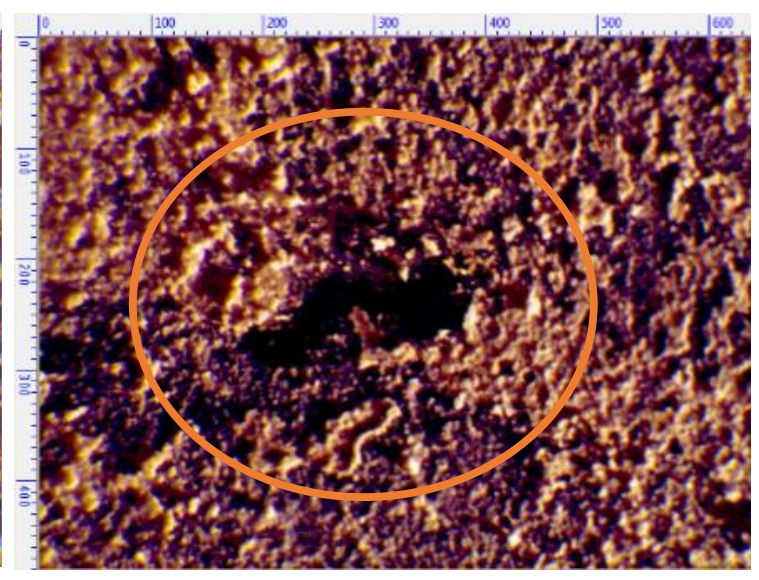

Figure 7.2: The same portion of material after lasing had been observed with damage. 


\section{B. Spectra and their Analysis}

\section{Spectra from ZnO Sample without Spheres}

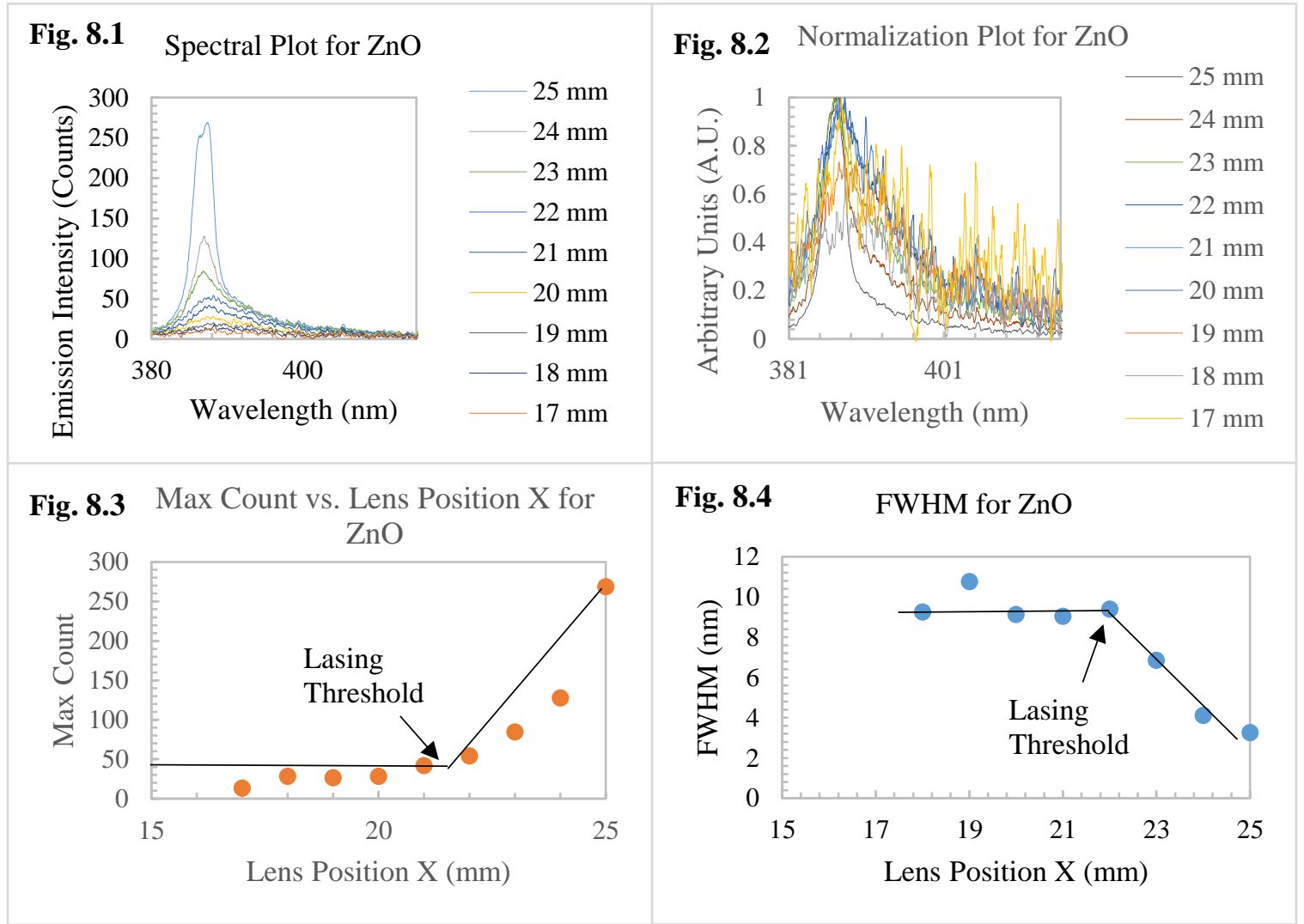

Figure 8.1: Spectra Plot for $\mathrm{ZnO}$ sample lasing after an increase in intensity with different lens distances. Figure 8.2: Normalization plot with different lens position.

Figure 8.3: Max Count vs. Lens Position X graph displaying lasing threshold.

Figure 8.4: FWHM plot of $\mathrm{ZnO}$.

Figures $8.1-8.4$ show data from a sample without spheres that was a multilayered $\mathrm{ZnO}$ film with $\mathrm{ZnO}$ particle size of $240 \mathrm{~nm}$. Figure 8.1 shows spectra of the $\mathrm{ZnO}$ sample transitioning from a non-lasing state to a lasing state. The top, blue spectrum was lasing with a linewidth of $3.33 \mathrm{~nm}$ FWHM at a lens position of $X=21.5 \mathrm{~mm}$, i.e. 8.5 $\mathrm{mm}$ from the focus position. The way we calculate the FWHM is from measuring half the height of our peak on both sides and subtracting both of the $\mathrm{x}$-values for the difference. 
The calculated wavelength on both sides of the peak was 385.21 and $388.54 \mathrm{~nm}$ with a difference of $3.33 \mathrm{~nm}$. The height of the spectrum was 263 counts and half of the max count being 131.5 counts. The peak is located at a wavelength of $386.9 \mathrm{~nm}$. A normalized plot of the spectra is shown in Figure 8.2 and displays light emission spectra for four different pump intensities. The normalization enables us to clearly see the difference in the widths of the spectra as the lens position changes. In Figure 8.3, the peak height is graphed against the pump intensity. As the pump intensity increases, the maximum count emitted from the sample increases superlinearly with the lens position at a threshold of $\sim 22 \mathrm{~mm}$. Figure 8.4, is a plot of the spectral linewidth of the emitted light. One notices a clear transition from PL with a linewidth of $\sim 10 \mathrm{~nm}$ to lasing with a much narrower linewidth of $\sim 3 \mathrm{~nm}$ FWHM. 


\section{Spectra of $\mathrm{ZnO}$ films with $83.3 \mathrm{~nm} \mathrm{SiO} 2$ Spheres}

\section{A. Spectral Analysis}

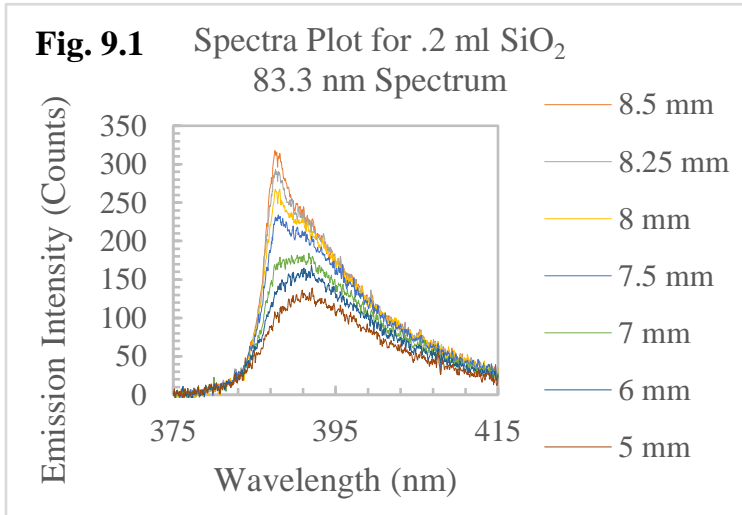

Fig. 9.2 Normalized Plot .2 $\mathrm{ml} \mathrm{SiO}_{2}$
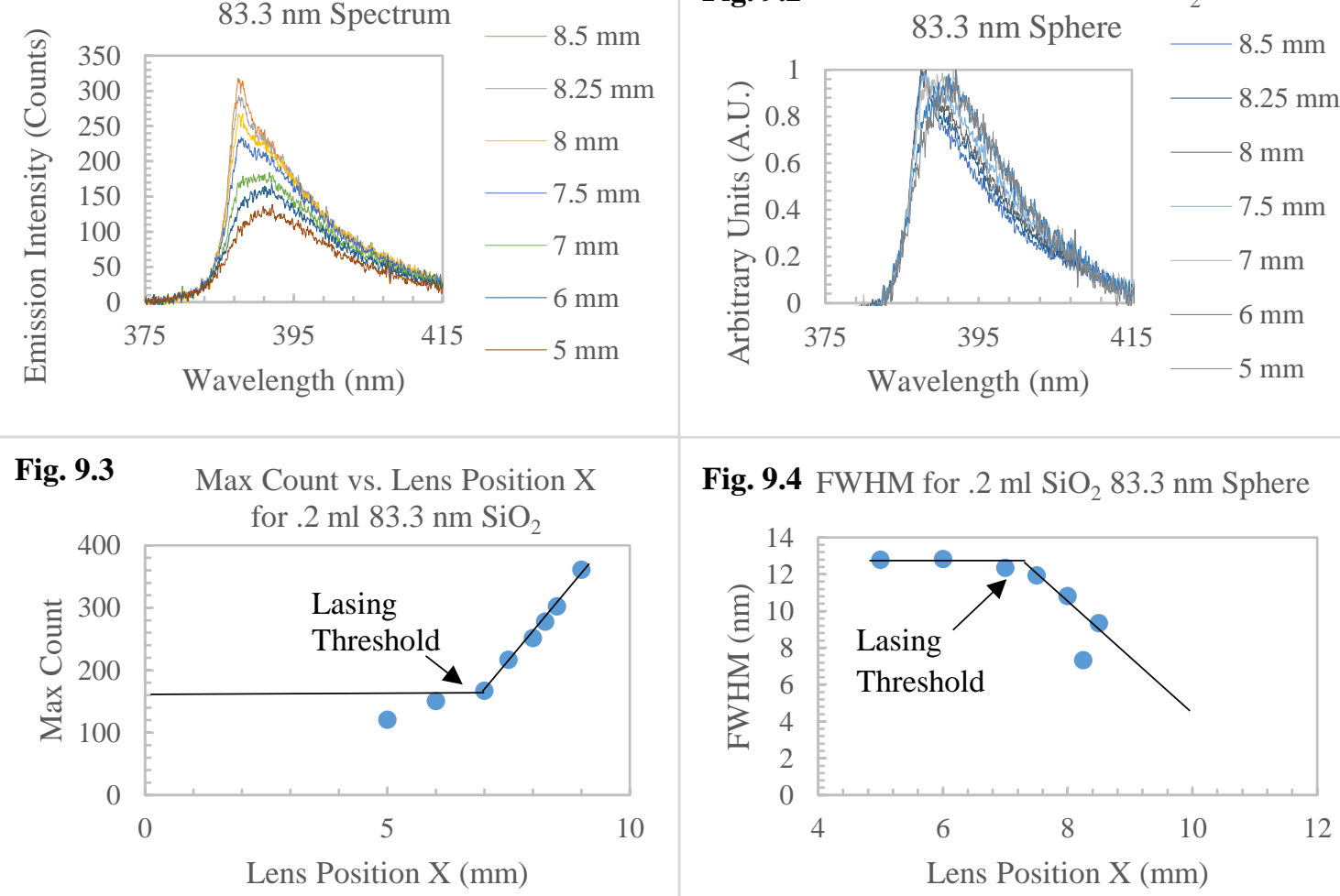

Fig. 9.4 FWHM for . $2 \mathrm{ml} \mathrm{SiO}_{2} 83.3 \mathrm{~nm}$ Sphere

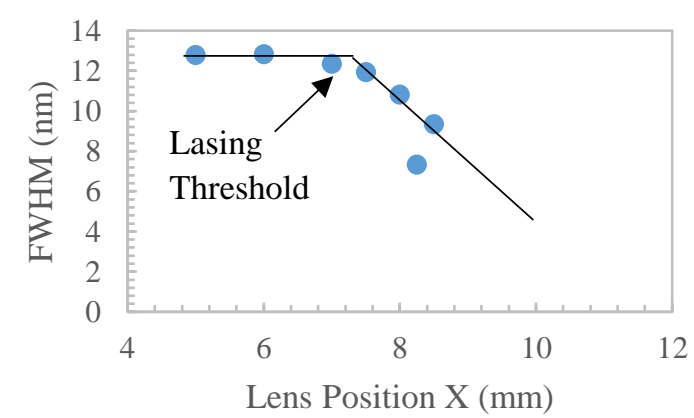

Figure 9.1: Spectra plot displaying a peak on the left-hand side as the intensity increases with an increase in distance.

Figure 9.2: Normalized spectra displayed for the collected distances

Figure 9.3: Threshold plot of the Max Count vs. Lens Position X.

Figure 9.4: FWHM data plot with lasing threshold labeled.

Figures $9.1-9.4$ show data from a sample that was a multilayered $\mathrm{ZnO}$ film with $\mathrm{SiO}_{2}$ spheres of $83.3 \mathrm{~nm}$ diameter and relative volume of $22 \%$. In Figure 9.1, we are able to see a narrow lasing peak emerge on the left-hand side at a wavelength of $387 \mathrm{~nm}$ as we move the focusing lens to a position of $7.5 \mathrm{~mm}$ and associate this narrow peak at a wavelength of $\lambda=387.1 \mathrm{~nm}$ with the emerging lasing line. At the same time, we see the total emitted light increase, even though the total number of incoming photons remains 
the same. This is a clear sign for an increase of the quantum efficiency as expected at the lasing threshold. In Figure 9.2, the spectral plots are normalized and the line narrowing, at FWHM, is observed. Figure 9.3 shows the signal intensity as a function of lens position. We applied guides for the eye for the first three data points representing the PL regime and the second set of five data points representing the lasing regime. The intersection is the lasing threshold which is found to be at a lens position of $7 \mathrm{~mm}$. Using eqs. (1) - (4) the pump intensity at the threshold is $4,358,591 \mathrm{~W} / \mathrm{mm}^{2}$. For the normalized spectra, Figure 9.2, we are able to see a decrease of the width of the spectra from $12 \mathrm{~nm}$ to $\sim 6 \mathrm{~nm}$. The threshold for the line narrowing in Fig. 9.4 occurs at a lens position of $\sim 7 \mathrm{~mm}$ which agrees reasonably well with the lasing threshold determined from Fig. 9.3.

Figures 10.1 - 10.4 graph data from a sample that was a multi-layered $\mathrm{ZnO}$ sample with $\mathrm{SiO}_{2}$ spheres with a diameter of $83.3 \mathrm{~nm}$ and relative volume a concentration of $36 \%$. Similar to Figure 9.1, we are able to see a peak emerging on the left-hand side of the spectrum in Figure 10.1. The peak wavelength is $388 \mathrm{~nm}$ at a lens position of $X=$ $11.1 \mathrm{~mm}$. For the strongest random lasing signal the linewidth FWHM is only $2.46 \mathrm{~nm}$ at $X=15.5 \mathrm{~mm}$. The normalized spectra plots in Figure 10.2 allow us to see the decrease in spectral width of the light emission spectra more clearly in comparison to smaller pump intensities. Figure 10.3 shows the threshold plot. The lasing threshold could not be precisely concluded from the data collected for the threshold plot. Since the collected data did not have any distinguishable, visible threshold we can only provide an upper limit for the threshold position of $\sim 7 \mathrm{~mm}$. The data points at large distances of $X$ are indicating there has been a transition into lasing with those larger three values FWHM 


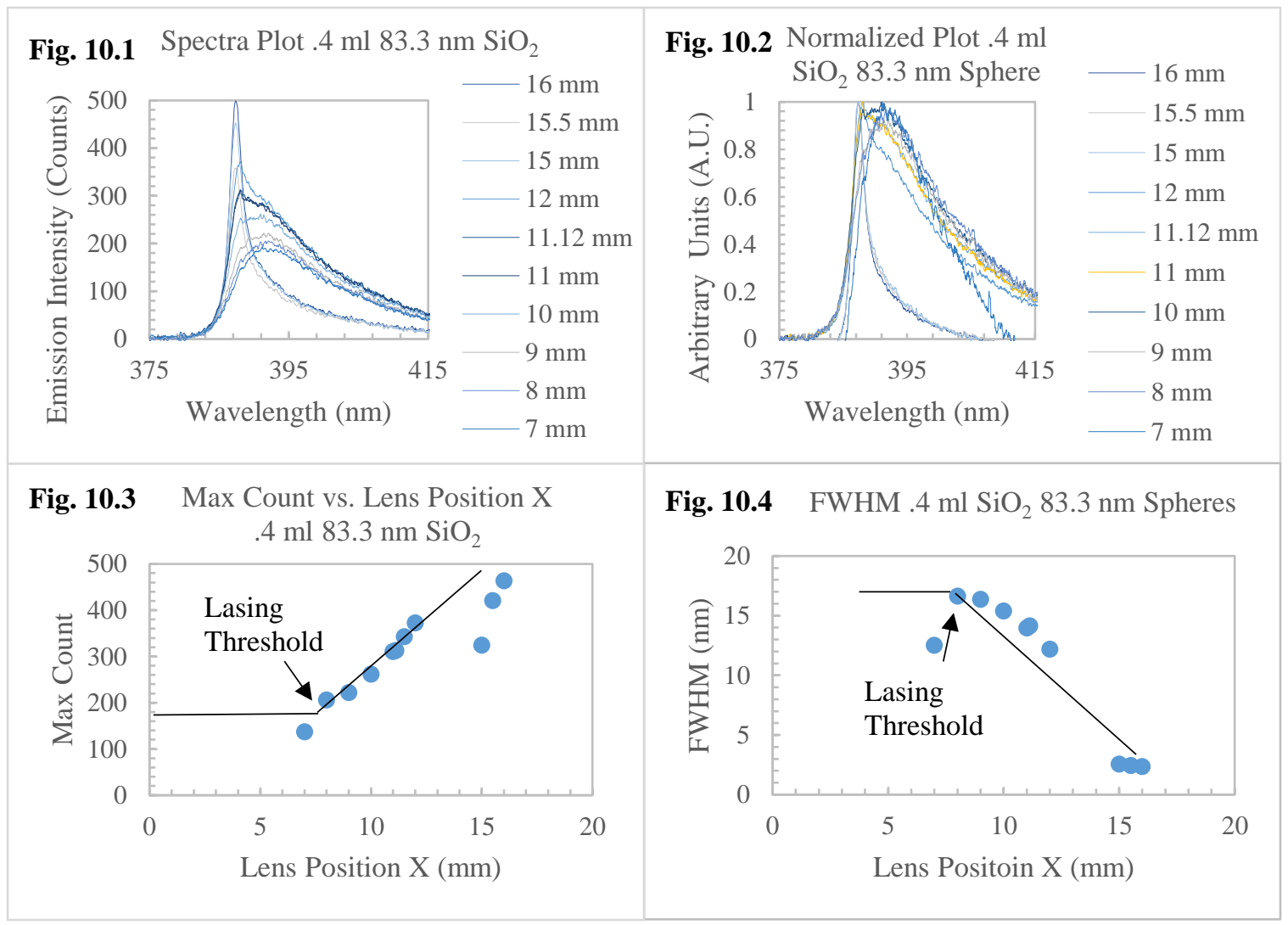

Figure 10.1: Spectra plot displaying a peak on the left-hand side as the intensity increases with an increase in distance.

Figure 10.2: Normalized spectra being displayed for the collected distances.

Figure 10.3: Plot of Max Count vs. Lens Position X.

Figure 10.4: FWHM plot for the $.4 \mathrm{ml} 83.3 \mathrm{~nm} \mathrm{SiO}_{2}$ sample.

being $\sim 2 \mathrm{~nm}$. In Figure 10.4 we have a FWHM plot indicating a transition from PL to lasing. The line-narrowing threshold is found to be at a lens position of $X=8 \mathrm{~mm}$, which is in reasonable agreement with the lasing threshold found in Fig. 10.3. There is only an $X$ difference of $1 \mathrm{~mm}$ between these two $X$ values for the figures.

We are able to conclude from the data collected that the sample did transition into a lasing state from a non-lasing state. Support is given by the observed line narrowing 
given by the FWHM measurements as pump intensity increases. This is also supported by the providing a threshold value of $X \cong 7 \mathrm{~mm}$.

Figures 11.1 - 11.4 graph data from a sample that was a multilayered $\mathrm{ZnO}$ sample with $\mathrm{SiO}_{2}$ spheres of diameter $83.3 \mathrm{~nm}$ with $.8 \mathrm{ml}$ nanosphere solution corresponding to a volume concentration of 53\%. Figure 11.1 show lasing observed throughout the whole pump intensity range. This behavior was double checked in several locations of the sample. We are able to see lasing characteristics in all of the spectra in Fig. 11.1, as each of the plots having a narrow peak emitted on the left-hand side of the spectra indicating where the $\mathrm{ZnO}$ lasing signal is located at. The peak positions in the spectra were consistently found at wavelengths of $387 \mathrm{~nm}$. Figure 11.2 shows normalized spectra that demonstrate the reduced linewidth and very small background of PL emission at higher pump intensities.

Figure 11.3 shows the threshold plot and indicates that there is an exceptionally low threshold for the sample. Since the lasing threshold is not distinguishable, we provide an upper limit for the threshold lens position of $X \cong 1-3 \mathrm{~mm}$. Figure 11.4 displays that the spectra line width reaches $2 \mathrm{~nm}$ at the highest pump intensities and a lasing threshold of $X$ $=3 \mathrm{~mm}$ which is in good agreement with Fig. 11.3. 


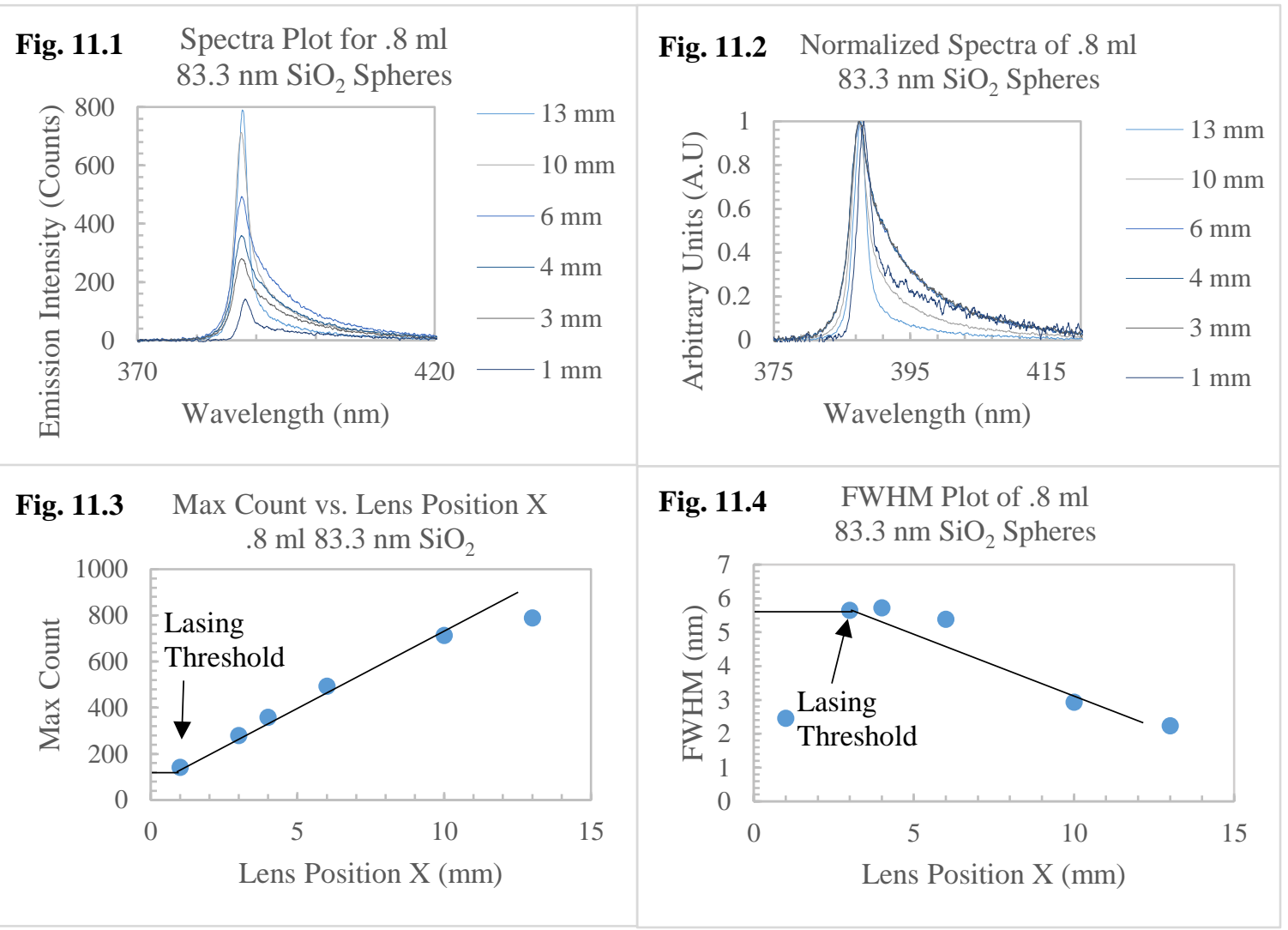

Figure 11.1: Spectra plot for $.8 \mathrm{ml} 83.3 \mathrm{~nm} \mathrm{SiO}{ }_{2}$ sample lasing with increase in intensity with increase in distances.

Figure 11.2: Normalized spectra displayed for the collected distances.

Figure 11.3: Displays the Max Count vs. Lens Position X graph with lasing threshold labeled.

Figure 11.4: FWHM with lasing threshold labeled. 
Figures 12.1 - 12.4 show data that was from a multilayered $\mathrm{SiO}_{2}$ sample with a diameter particle size of $83.3 \mathrm{~nm}$ with $2 \mathrm{ml}$ nanosphere solution with a volume concentration of $73 \%$. In Figure 12.1, we are able to see a peak emerging from the lefthand side of the spectra between $387.74-388.3 \mathrm{~nm}$ as the pump intensity increases. The normalized spectra in Figure 12.2 demonstrate a decrease in the width of the spectra when increasing the pump intensity. Figure 12.3 shows the threshold plot. This plot does not have a distinguishably clear lasing threshold, we provide the upper limit for the threshold position that is reached at $X \cong 7 \mathrm{~mm}$. In Figure 12.4, we display the linewidth reduction in the transition from PL to lasing. The values line-narrowing threshold is found to be at $X=8 \mathrm{~mm}$. We are able to determine that there is again lasing with spectral peaks between the wavelengths of $387.74-388.3 \mathrm{~nm}$.

Summarizing the results for $\mathrm{ZnO}$ films with $83.3 \mathrm{~nm}$ spheres, we find that the $\mathrm{ZnO}$ films with $83.3 \mathrm{~nm} \mathrm{SiO}_{2}$ spheres perform consistently better than pure $\mathrm{ZnO}$ films: In films with $83.3 \mathrm{~nm}$ spheres the lasing thresholds are reduced and narrower laser lines can be achieved. The improvement appears to increase with higher sphere concentrations up to $53 \%$ relative sphere volume. In the films with relative sphere volume of $73 \%$ the lasing parameters are still good but appear to be slightly inferior to those in films with $53 \%$ sphere volume. 
Fig. 12.1 Spectra Plot $2 \mathrm{ml} 83.3 \mathrm{~nm} \mathrm{SiO}_{2}$ Spheres

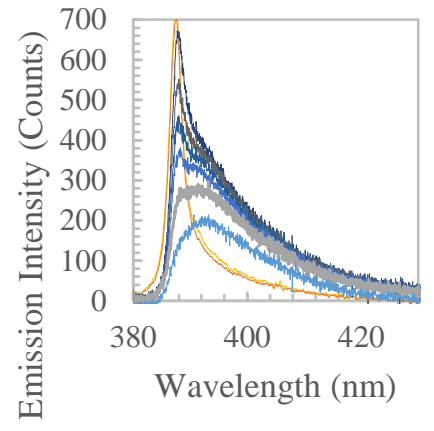

Fig. 12.3 Max Count vs. Lens Position X $2 \mathrm{ml} 83.3 \mathrm{~nm} \mathrm{SiO}_{2}$

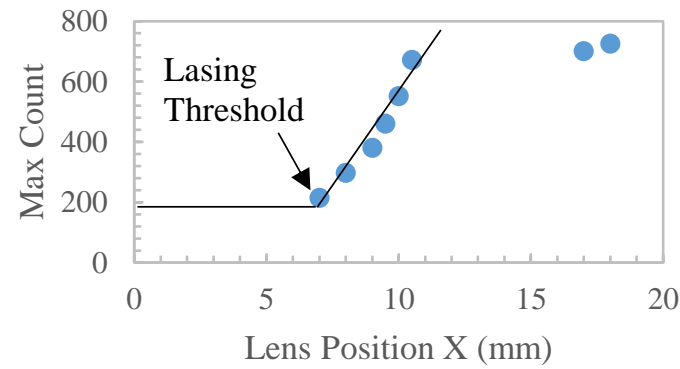

Fig. 12.2 Normalization Spectra for $2 \mathrm{ml}$ $83.3 \mathrm{~nm} \mathrm{SiO} 2$ Spheres

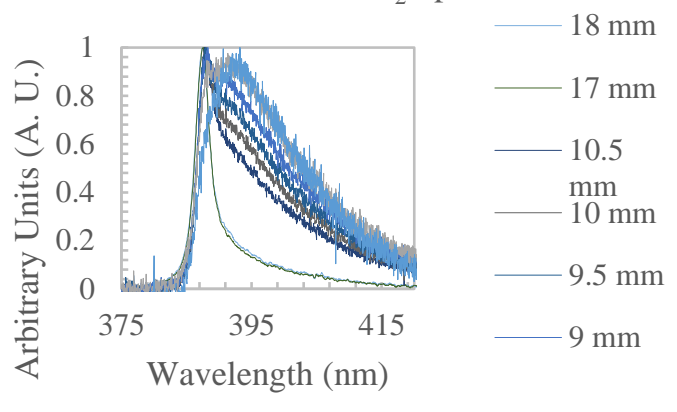

Fig. 12.4 FWHM $2 \mathrm{ml} \mathrm{SiO} 283.3$ nm Sample

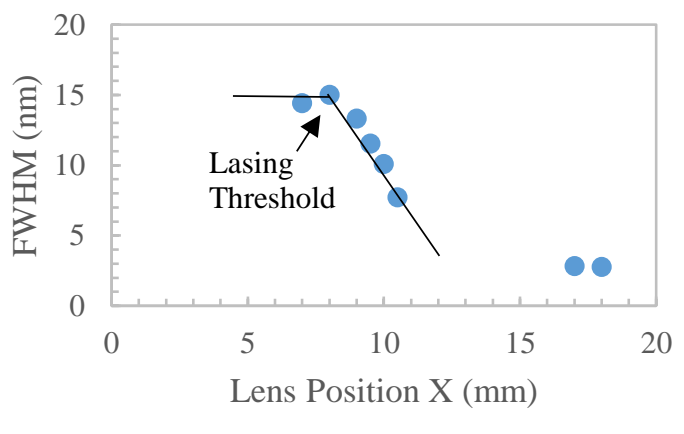

Figure 12.1: Spectra Plot of Emission Intensity vs. Wavelength.

Figure 12.2: Normalized graph of all the spectra plots.

Figure 12.3: Threshold plot with Max Count vs. Lens Position X.

Figure 12.4: FWHM plot with lasing threshold indicated. 


\section{3. $\mathrm{ZnO}$ films with $172.5 \mathrm{~nm} \mathrm{SiO} 2$ Spheres}

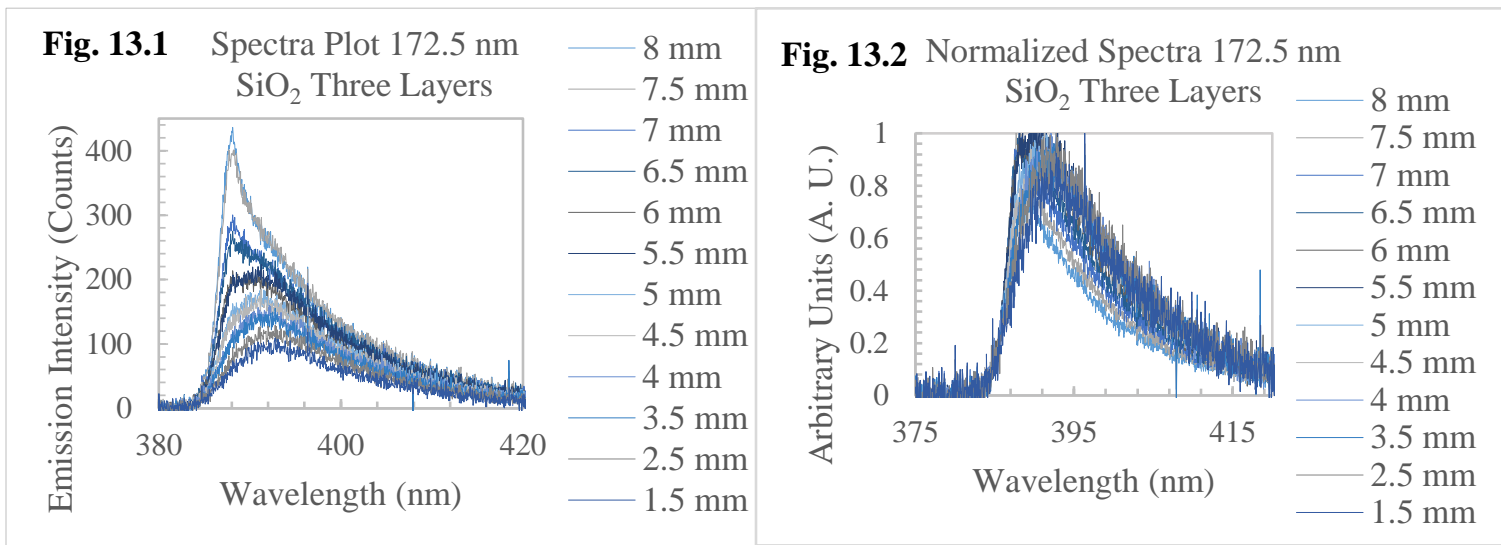

Fig. 13.3 Max Count vs. Lens Position X $172.5 \mathrm{~nm} \mathrm{SiO} 2$ Three Layers

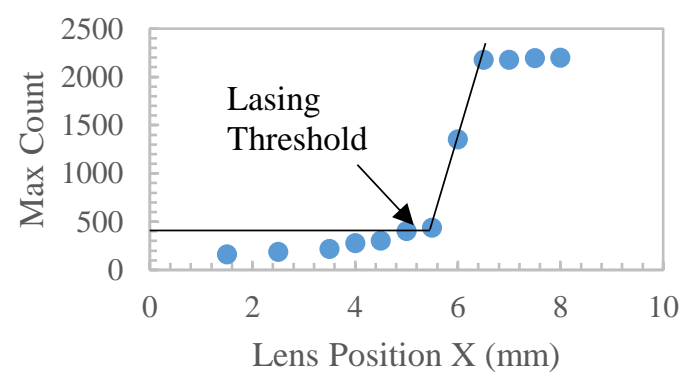

Fig. 13.4 FWHM $172.5 \mathrm{~nm} \mathrm{SiO} 2$ Three Layers

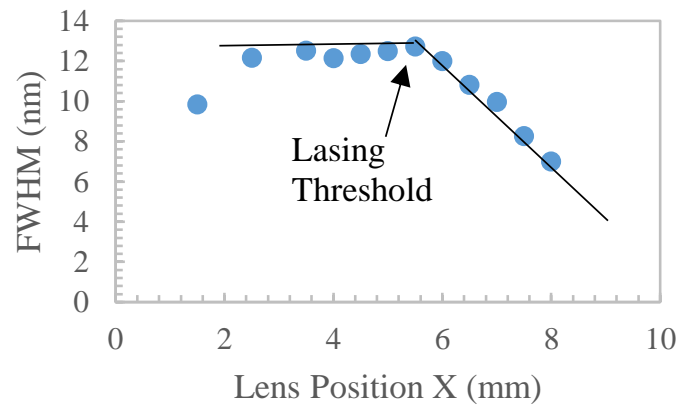

Figure 13.1: A spectra plot of Emission Intensity vs. Wavelength. Figure 13.2: Normalized spectra graph of increasing distances. Figure 13.3: Threshold plot with Max Count vs. Lens Position X. Figure 13.4: FWHM plot with lasing threshold labeled.

Figures 13.1 - 13.4 show data from a three layered $\mathrm{ZnO}$ sample with $\mathrm{SiO}_{2}$ spheres of diameter $172.5 \mathrm{~nm}$ with $.4 \mathrm{ml}$ nanosphere solution with a relative sphere volume of 36\%. What we are seeing in Figure 13.1 is an emerging laser peak on the left-hand side of the spectra as we are increasing the pump intensity. The peak appears at $388.15 \mathrm{~nm}$. In Figure 13.2, we see from the normalized spectra a decrease in the spectral width as the lens position $X$ increases.

The threshold plot of Fig. 13.3, has a flat portion up to a lens position of $X \sim 5 \mathrm{~mm}$, 
then a steep increase and a plateau at a lens position of $7 \mathrm{~mm}$. The spectra shown in Fig. 13.1 indicate the sample is still in PL from 1.5 - $5.5 \mathrm{~mm}$ but transitions from non-lasing to a lasing state at $X=6 \mathrm{~mm}$. The plateauing for the top group of data points was related to the damage that the surface of the sample was receiving at the time from high pump intensities, resulting in the sample being burned off the substrate.

The normalized spectra in Fig. 13.2 as well as Fig. 13.4 show that the width of the spectra continuously decreases indicating that lasing is maintained even for the damaged sample area. 
Fig. 14.1 Spectra Plot $172.5 \mathrm{~nm} \mathrm{SiO}_{2}$

Spheres One Layer

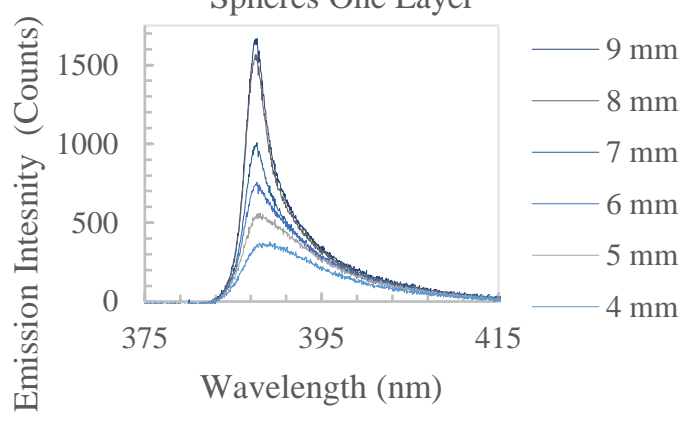

Fig. 14.3 Max Count vs. Lens Position X $172.5 \mathrm{~nm} \mathrm{SiO} 2$ One Layer

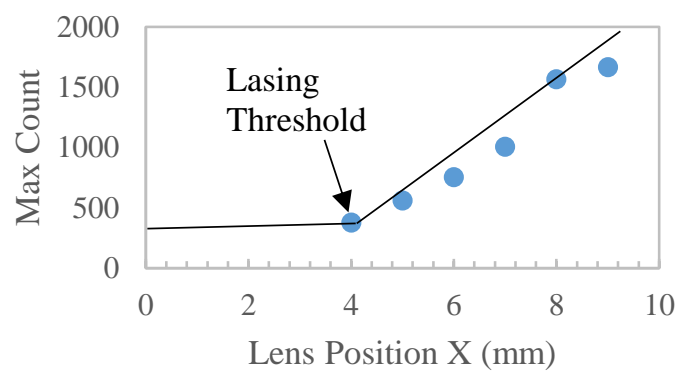

Fig. 14.2 Normalized Spectra $172.5 \mathrm{~nm}$

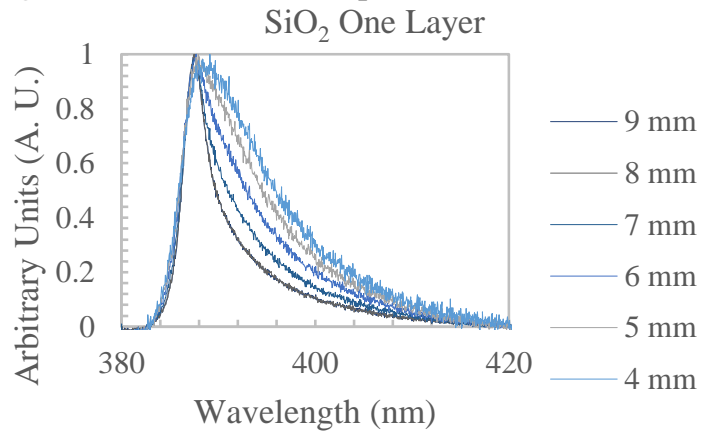

Fig. 14.4 FWHM $172.5 \mathrm{~nm} \mathrm{SiO}_{2}$ One Layer

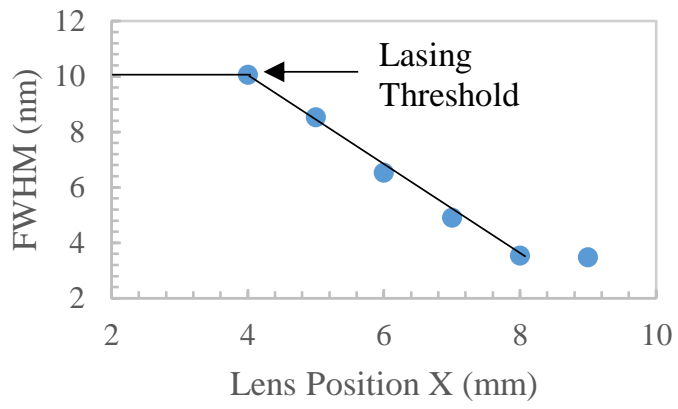

Figure 14.1: A spectra plot of Emission Intensity vs. Wavelength.

Figure 14.2: Normalized spectra graph of increasing distances.

Figure 14.3: A Max Count vs. Lens Position $X$ displaying the lasing threshold.

Figure 14.4: FWHM plot with lasing threshold labeled.

Figures 14.1 - 14.4 show data from a sample that was a single layer of $\mathrm{SiO}_{2} \mathrm{ZnO}$ with a sphere diameter $172.5 \mathrm{~nm}$ and prepared from a $.4 \mathrm{ml}$ nanosphere solution with a volume concentration of $36 \%$. A lasing peak is appearing with a maximum at a wavelength of $387.7 \mathrm{~nm}$. We are able to see the spectra going very smoothly from nonlasing to lasing and it is difficult to determine a clear threshold. From the data of Figs. 14.3 and 14.4 only an upper limit for the lasing threshold position can be determined i.e. the $X_{T H}<4 \mathrm{~mm}$. 
The normalized spectra in, Figure 14.2, also demonstrate that the width of the spectra decreases continuously and smoothly at lens positions of $X=4 \mathrm{~mm}$ or larger.

\section{Summary for pure $\mathrm{ZnO}$ films and $\mathrm{ZnO}$ films with $\mathrm{SiO}_{2}$ Spheres}

Figure 15.1 presents a summary of all seven threshold graphs of the samples discussed so far and Figure 15.2 has all of the linewidth data plotted for the seven samples. It is found that the samples with spheres have a lower lasing threshold than pure $\mathrm{ZnO}$ films.

We find that the lowest lasing thresholds and the strongest line narrowing occur in films with $.8 \mathrm{ml} 83.3 \mathrm{~nm} \mathrm{SiO} 2$ spheres having relative sphere volume of $\sim 53 \%$. This impression is confirmed in Table 1 that summarizes the data in numerical form and in Fig. 16.1 columns 1 and 2 from Table 1 are displayed. 
Fig. 15.1 Max Count vs. Lens Position X for all 7 samples

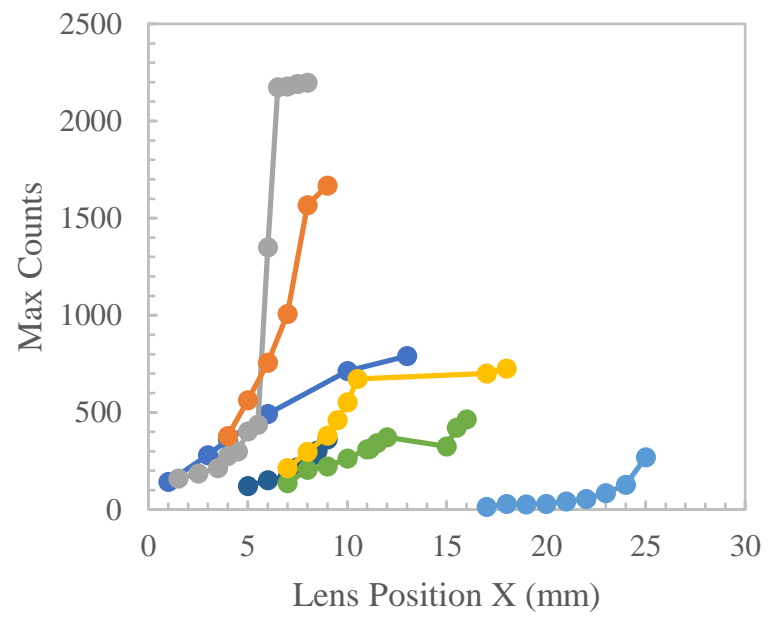

$-\mathrm{ZnO}$

$\longrightarrow .2 \mathrm{ml} 83.3 \mathrm{~nm}$

$-.4 \mathrm{ml} 83.3 \mathrm{~nm}$

$-8 \mathrm{ml} 83.3 \mathrm{~nm}$

$-2 \mathrm{ml} 83.3 \mathrm{~nm}$

$-172.5 \mathrm{~nm}$ Three Layers

- 172.5 nm One Layer

Fig. 15.2 Linewidths (FWHM) of All Seven Samples
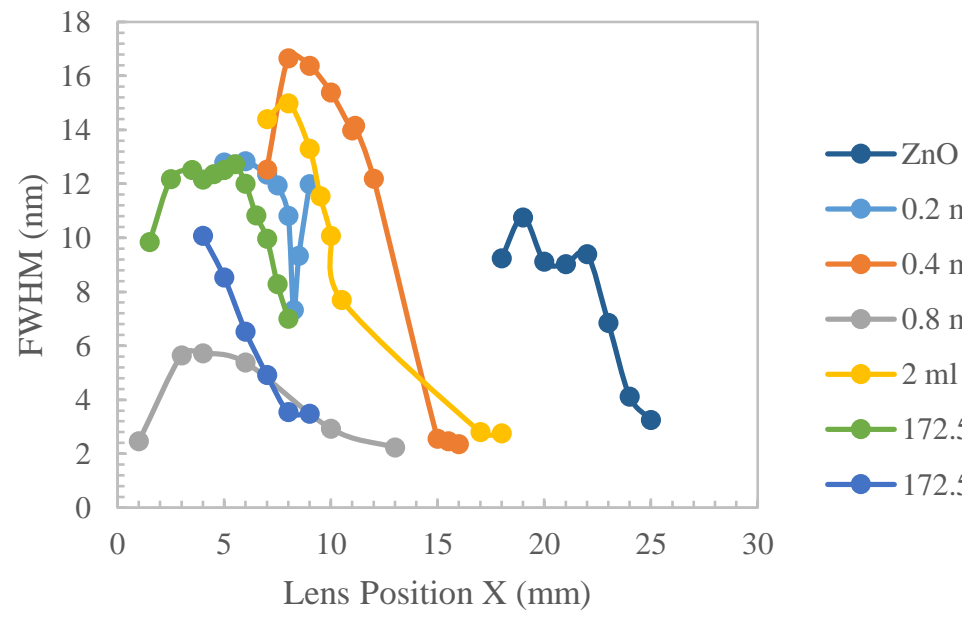

$-0.2 \mathrm{ml} 83.3 \mathrm{~nm}$

$-0.4 \mathrm{ml} 83.3 \mathrm{~nm}$

$-0.8 \mathrm{ml} 83.3 \mathrm{~nm}$

$-2 \mathrm{ml} 83.3 \mathrm{~nm}$

$-172.5 \mathrm{~nm}$ Three Layer

$\longrightarrow 172.5$ nm One Layer

Figure 15.1: Threshold plot with Max Count vs. Lens Position $X$ for all seven samples. Figure 15.2: FWHM plot for the seven samples. 


\begin{tabular}{|c|c|c|c|c|}
\hline Sample & $\begin{array}{c}\text { Lasing } \\
\text { Threshold } \\
(\mathbf{m m})\end{array}$ & $\begin{array}{c}\text { Line Narrowing } \\
\text { Threshold (mm) }\end{array}$ & $\begin{array}{c}\text { Smallest } \\
\text { Linewidth } \\
\text { Measured (nm) }\end{array}$ & $\begin{array}{c}\boldsymbol{R S V}_{\text {Film }} \\
\text { (\%) }\end{array}$ \\
\hline $\mathrm{ZnO}$ & 23 & 23.4 & 3.26 & \\
\hline $\begin{array}{c}83.3 \mathrm{~nm} .2 \\
\mathrm{ml}\end{array}$ & 7 & 9.8 & 7.33 & 22 \\
\hline $\begin{array}{c}83.3 \mathrm{~nm} .4 \\
\mathrm{ml}\end{array}$ & 7 & 11.8 & 2.35 & 36 \\
\hline $\begin{array}{c}83.3 \mathrm{~nm} .8 \\
\mathrm{ml}\end{array}$ & $1-3$ & 4 & 2.23 & 53 \\
\hline $83.3 \mathrm{~nm} 2 \mathrm{ml}$ & 7 & 10.4 & 2.32 & 73 \\
\hline $\begin{array}{c}172.5 \mathrm{~nm} .4 \\
\mathrm{ml} 1 \text { Layer }\end{array}$ & 4 & 6.4 & 3.49 & 36 \\
\hline $\begin{array}{c}172.5 \mathrm{~nm} .4 \\
\mathrm{ml} 3 \mathrm{Layer}\end{array}$ & 5.5 & 8.4 & 7 & 36 \\
\hline
\end{tabular}

Table 1: The table displays the values of the lasing threshold, line narrowing threshold, smallest linewidth measured, and $R S V_{\text {Film }}$.

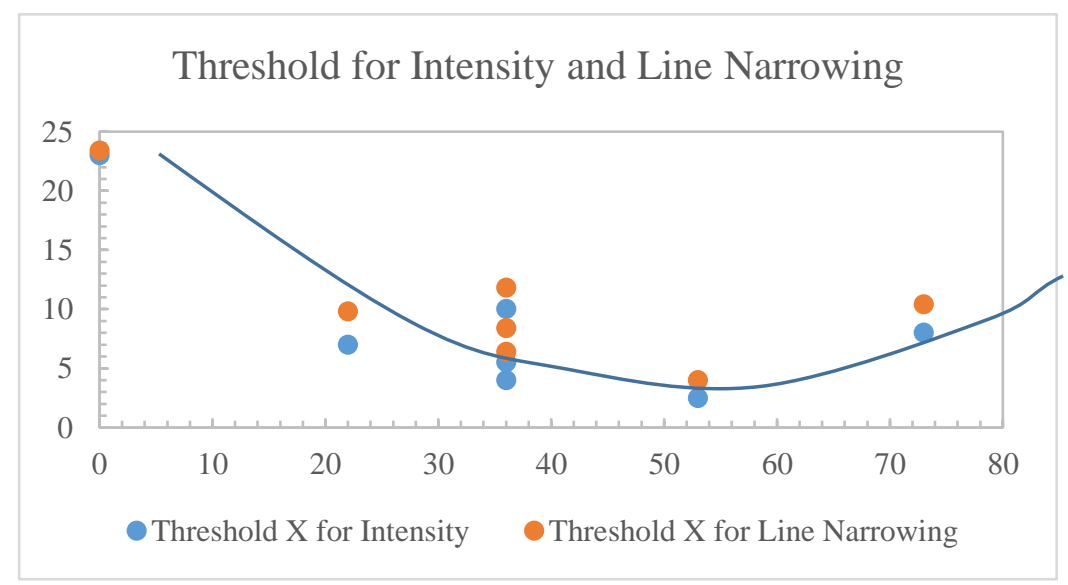

Fig. 16.1: The graph displays the threshold $X$ for intensity and line narrowing.

We thus conclude from table and graph that the best random lasing properties in our films are obtained for $R S V_{\text {Film }} \approx 50 \%$. 


\section{Spectra from samples with Latex Spheres}

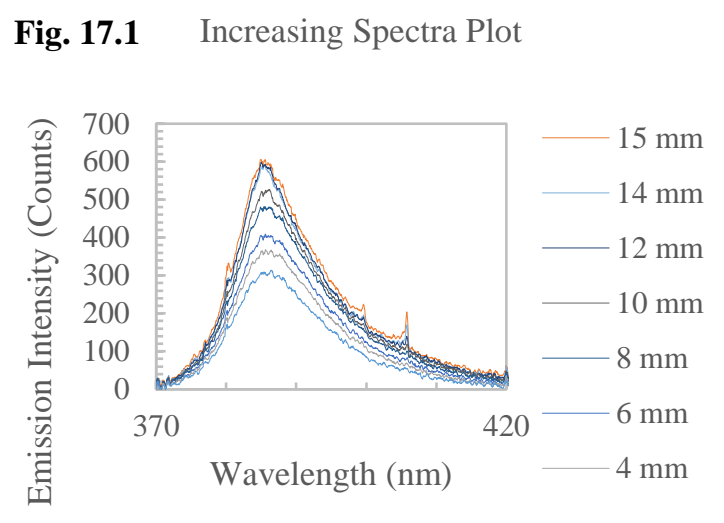

Fig. 17.3 Single Spectrum Plot

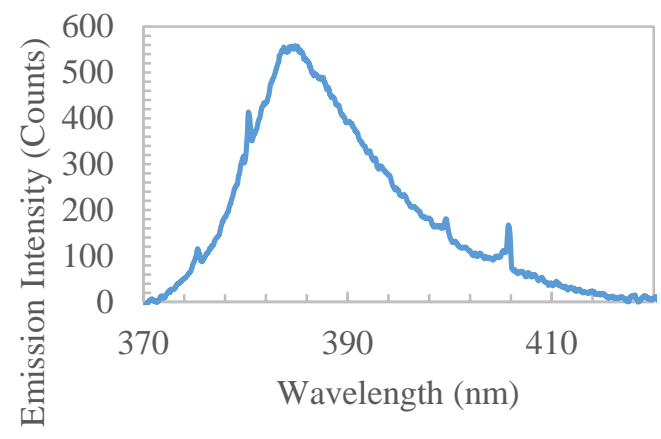

Fig. 17.2 Decreasing Spectra Plot

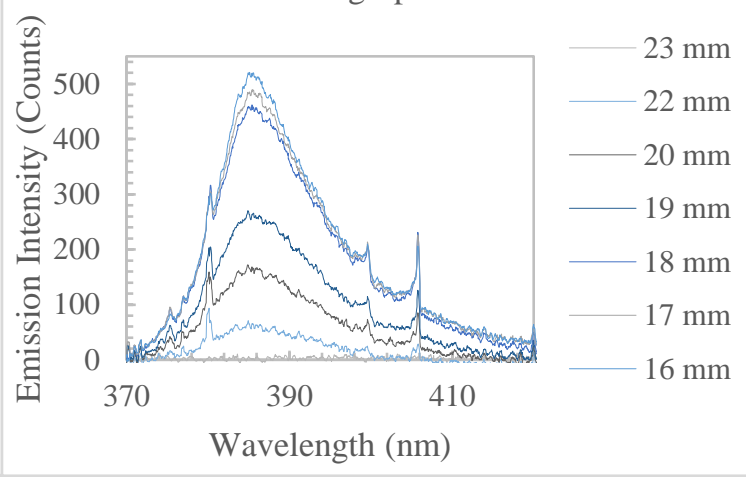

Fig. 17.4 Lens Position X vs. Peak Height

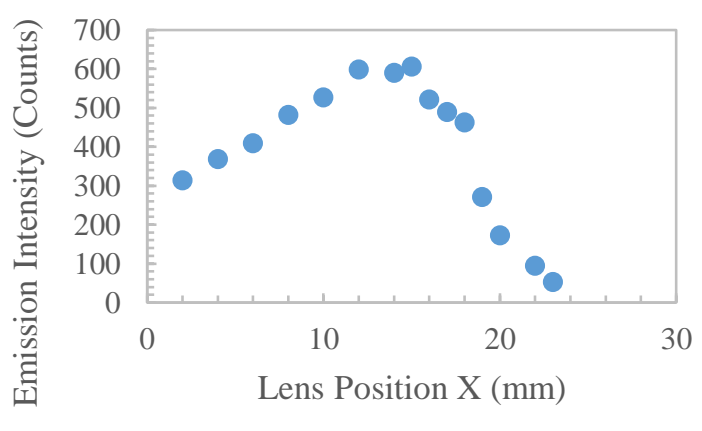

Fig. 17.5 FWHM for Latex spheres

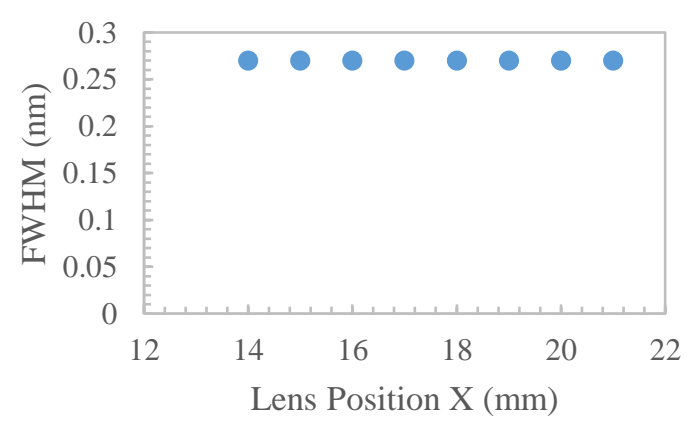

Figure 17.1: Increasing spectra plot for the Latex sphere sample.

Figure 17.2: Decreasing spectra plot for the Latex sphere sample.

Figure 17.3: Displays one spectrum plot to emphasize four peaks appearing on the sides of the gaussian peak that are located at $375.31,380.28,399.65$, and $405.78 \mathrm{~nm}$.

Figure 17.4: The graph displays the change in Emission Intensity when the Pump Intensity is increased.

Figure 17.5: Linewidth plot for the Latex sphere sample for the narrow line at $405.78 \mathrm{~nm}$ peak. 
The following results relate to the $\mathrm{ZnO}$ films with Latex spheres of particle diameter $.86 \mu \mathrm{m}$. These results are displayed in Figs. 17.1-17.5. The sample went through a similar sample preparation as the samples discussed earlier and the $\mathrm{ZnO}$ was also annealed at $550{ }^{\circ} \mathrm{C}$.

Figure 17.1 shows spectra obtained over a large range of pump intensities. The behavior in these spectra is different from the results described earlier. We see in Fig. 17.1 that the spectra show only small changes in overall shape and amplitude as the pump intensity is increased. Even at high pump intensities there seems to be no significant line narrowing, that would indicate a transition from PL to lasing. We also only observe a small increase of the light emission from the sample as the lens position $X$ is increased from $X=0$ to $X=15 \mathrm{~mm}$ confirming the lack of lasing. Figs. 17.2 and 17.3 even show a decreasing light emission from the sample as $X$ is increased beyond $15 \mathrm{~mm}$. We could confirm that this decrease is caused by the sample being burned off the substrate and there being less of a sample for the pump laser to interact with. There is, hence, no lasing threshold as we saw in all other films, and sample damage appears to occur at lower pump intensities than seen before.

Fig. 17.5 displays the linewidths for the narrow line at $405.7 \mathrm{~nm}$ at different lens positions. What is expected when analyzing FWHM is that there should be a change in FWHM when the pump laser spot size changes. The data should display a larger FWHM with lower intensities and a smaller FWHM at higher intensities. However, each FWHM is the same, indicating there is no transition for the sample from non-lasing to lasing. 
Another difference to the earlier results is the appearance of four very narrow peaks in the spectra in Fig. 17.3. Such peaks had not been observed in any of the other films. The peaks appear at a lens position of $X=10 \mathrm{~mm}$ and are seen to increase slightly with the pump intensity. The peaks in our spectra appear at wavelengths of 375.31 , $380.28,399.65$, and $405.78 \mathrm{~nm}$. Two peaks are seen on each side of the spectra maximum.

To explain these new peaks, we present data from a detailed description of the $\mathrm{N}_{2}$

Fig. 17.6

\begin{tabular}{ccc}
380.49 & $\leq 2.0$ & $\begin{array}{c}(0,2) \text { band of } C^{3} \pi_{u}-B^{3} \pi_{g} \mathrm{~N}_{2} \\
\text { second positive system }\end{array}$ \\
405.94 & $<2.0$ & $\begin{array}{c}(0,3) \text { band of } C^{3} \pi_{u}-B^{3} \pi_{g} \mathrm{~N}_{2} \\
\text { second positive system }\end{array}$ \\
375.54 & $<2.0$ & $\begin{array}{c}(1,3) \text { band of } C^{3} \pi_{u}-B^{3} \pi_{g} \mathrm{~N}_{2} \\
\text { second positive system }\end{array}$ \\
399.84 & $<2.0$ & $\begin{array}{c}(1,4) \text { band of } C^{3} \pi_{u}-B^{3} \pi_{g} \mathrm{~N}_{2} \\
\text { second positive system }\end{array}$ \\
353.67 & $<2.0$ & $\begin{array}{c}(1,2) \text { band of } C^{3} \pi_{u}-B^{3} \pi_{g} \mathrm{~N}_{2} \\
\text { second positive system }\end{array}$ \\
\hline
\end{tabular}

Fig. 17.7

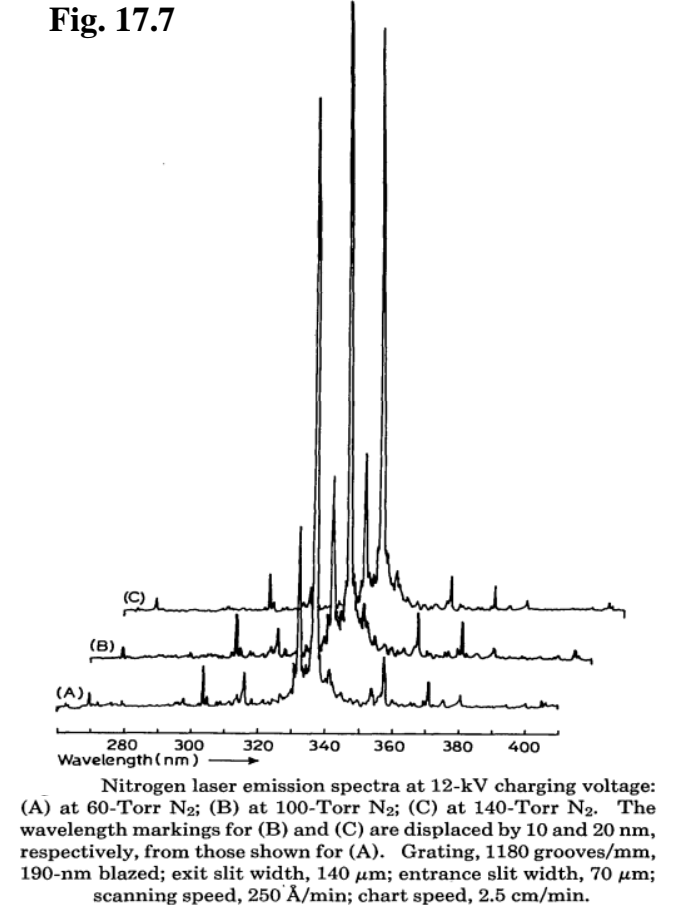

Figure 17.6: A table of known $\mathrm{N}_{2}$ lasing wavelengths.

Figure 17.7: Nitrogen laser emission spectra.

Note: Figures 17.6 and 17.7 were taken from the article [34] Subhash, N., Kartha, S. C., and Sathianandan, K. "New Vibrational Bands in Nitrogen Laser Emission Spectra." Applied Optics. vol. 22, pp. 3612-3617 (1983).

laser spectrum taken from a paper by N. Subhash et al. in ref. [34]. Fig. 17.6 displays 
known $\mathrm{N}_{2}$ lasing wavelengths and Fig. 17.7 shows the complete line spectrum of the nitrogen laser. Apart from the main line at $337.1 \mathrm{~nm}$ there are a number of less intense lines. The most prominent of these are listed to be at 380.49, 405.94, 375.54, 399.84 and $353.67 \mathrm{~nm}$. A comparison shows that the four new lines in our spectrum are identical to the listed $\mathrm{N}_{2}$ lasing sidelines. The $5^{\text {th }} \mathrm{N} 2$ line at $353.67 \mathrm{~nm}$ is outside our spectral range and was thus unobservable for us.

This finding indicates that the burn-off observed takes place at lens positions $X>$ $15 \mathrm{~mm}$ opened a window for the very weak N2 lasing sideline such that they were transmitted through the sample and recorded in our spectrometer. We attribute the burnoff of the $\mathrm{ZnO} /$ nanosphere film to disintegration of the Latex spheres under pumping. The polystyrene melting point is at $250{ }^{\circ} \mathrm{C}$ and the glass transition temperature at around $100{ }^{\circ} \mathrm{C}$. It appears very likely that during the optical pumping these temperature limits were reached leading to the disintegration and destruction of the film structure.

Besides these findings, the published spectrum for the $\mathrm{N}_{2}$ laser also confirms that calibration of our spectrometer is reliable and good since the data we were collecting are very nearly identical to the published data. The spectrum in Fig. 17.5 has peaks with a linewidth of $\sim 1 \mathrm{~nm}$ FWHM which is also consistent with our estimated wavelength resolution of $.7 \mathrm{~nm}$. 


\section{Summary and Conclusion}

Nano-particle $\mathrm{ZnO}$ films were prepared from sub-micron $\mathrm{ZnO}$ crystallites and studied for PL and RLg. Sample sets consisting of pure $\mathrm{ZnO}$ films as well as $\mathrm{ZnO}$ films with added $\mathrm{SiO}_{2}$ spheres, and one sample consisting of a $\mathrm{ZnO}$ film with Latex spheres were made by first annealing the $\mathrm{ZnO}$ at $550{ }^{\circ} \mathrm{C}$, and subsequently preparing liquid suspensions of spheres, and $\mathrm{ZnO}$-particles. This was followed by drop and dip coating, and subsequent drying of the layers at $100{ }^{\circ} \mathrm{C}$. An apparatus consisting of a pulsed $\mathrm{N}_{2}$ laser, focusing lens, a microscope, and a digital spectrometer was used to obtain light emission spectra under a variety of excitation conditions.

It was found that all samples showed $\mathrm{ZnO} \mathrm{PL}$ with peak wavelengths between $380 \mathrm{~nm}$ and $395 \mathrm{~nm}$ and a spectral width of 10 - $13 \mathrm{~nm}$ FWHM. Pure ZnO films as well as samples with $\mathrm{SiO}_{2}$ spheres showed line-narrowing and strong threshold-like increases in light emission at higher pump intensities. The threshold behavior was attributed to the onset of RLg. At the same time a narrowing of the emission linewidth was observed supporting the idea of a lasing transition. Lasing line widths as small as $2 \mathrm{~nm}$, i.e. significantly below the PL linewidth in $\mathrm{ZnO}$ were recorded.

Samples of pure $\mathrm{ZnO}$ showed qualitatively similar behavior, but the line narrowing and the threshold-type light emission behavior required somewhat higher pump intensities than in the $\mathrm{ZnO}$ films with built-in $\mathrm{SiO}_{2}$ nanospheres. A sample with Latex spheres showed no line narrowing and no threshold behavior, but the emission spectrum had 4 very narrow lasing lines. These lasing lines were identified as being sidelines of the $\mathrm{N}_{2}$ pump laser. It was concluded that the Latex spheres in this sample 
might have become damaged in the measuring process and that pump light from the $\mathrm{N}_{2}$ laser was transmitted through the sample, when the sample was damaged and burned off in the pumping.

All data allow the conclusion that the addition of $\mathrm{SiO}_{2}$ spheres to the $\mathrm{ZnO}$ powder films improve the RLg in the films. The observed improvement is likely due to advantageous changes of the scattering in the films. Adding transparent material may increase the scattering length and hence feedback.

The best sample among the set investigated had $\mathrm{SiO}_{2}$ spheres with $83.3 \mathrm{~nm}$ diameter and relative volume of 53\%. These $\mathrm{ZnO} /$ sphere films showed a lasing line width of $2.5 \mathrm{~nm}$, a threshold of $\sim 3 \mathrm{MW} / \mathrm{mm}^{2}$ for 800 ps pulses with a lasing intensity that was about 2 orders of magnitude higher than the pure $\mathrm{ZnO}$ sample.

Overall, we find that these data look promising for the continued research of nanospheres and their effect on RLg in particle films. $\mathrm{SiO}_{2}$ spheres have sufficiently high melting points, will remain intact in sample anneal procedures, and in operation with high pump intensities. 


\section{References}

[1] Verdeyen, Joseph T. Laser Electronics. (2 ${ }^{\text {nd }}$ ed). Englewood Cliffs, NJ: Prentice Hall. 1989.

[2] Pierret, Robert F. Semiconductor Device Fundamentals with Computer-Based Exercises and Homework Problems. (11 ${ }^{\text {th }}$ ed.). New York, NY: Pearson Education, Inc. 2015.

[3] Wiersma, Diederik. “The Smallest Random Laser.” Laser Physics. vol. 406, pp. 132 33 (2000).

[4] "Random Laser.” Wikipedia. 2018. http://en.m.wikipedia.org/wiki/Random_Laser

[5] Redding, B., Choma, M. A., and Cao, Hui. "Speckle-free laser imaging using random laser illumination." Nature Photonics. vol. 6, pp. 355-359 (2012).

[6] Myers, R. A. and Dixon, R. W. "Who invented the laser: An Analysis of the Early Patents.” Historic Studies in the Natural Sciences. vol. 34, pp. 115-149 (2003). http://hsns.ucpress.edu/content/34/1/115.full.pdf+html.

[7] Baklykin, Victor I. "The Scientific Career of VS Letokhov." IOPScience. vol. 85, pp. 5 (2012). http://iopscience.iop.org/article/10.1088/0031-8949/85/05/050302/meta

[8] Fujimura, R., Nagai, Y., and Kajikawa, K. "Coherent Random Laser Fluid of Nematic Liquid Crystal Emulsions." Japanese Journal of Applied Physics vol. 53, pp. 248002.1- 248002.5, (2013). http://iopscience.iop.org/article/10.7567/JJAP.53.01AE05.

[9] Schirber, Michael. “Synopsis: Shaking Up a Grain-Based Laser.” Physics Review Letter. vol. 108, pp. 248002.1-5 (2012). http://journals.aps.org/prl/abstract/10.1103/PhysrevLet.108.248002.

[10] Chang, Shu-Wei. “A White Random Laser.” Scientific Reports. vol. 8, pp. 2720.1 10 (2018). http://europepmc.org/article/PMC/5807428.

[11] Srikant, V., and Clarke, D. R. "On the optical band gap of zinc oxide.” Journal of Applied Physics. vol. 83, pp. 5447 (1998). http://doi.org/10.1063/1.367675.

[12] W. Shan, W. Walukiewicz, J. W. Ager III, and K. M. Yu, H. B. et al. "Nature of room-temperature photoluminescence in ZnO." Applied Physics Letter. vol. 86, pp. 191911 (2005). http://Nature of room-temperature photoluminescence in $\mathrm{ZnO}$ : Applied Physics Letters: Vol 86, No 19 (scitation.org).

[13] “Exciton.” Wikipedia. 2018. https://en.wikipedia.org/wiki/Exciton. 
[14] Sun, Y., Ketterson, J. B., and Wong, G. K. L., "Excitonic gain and stimulated ultraviolet emission in nanocrystalline zinc-oxide powder." Applied Physics Letter. vol. 77, pp. 2322-2324 (2000). http://doi.org/10.1063/1.1316069.

[15] Cao, H. and Zhao, Y. G. "Random Laser Action in Semiconductor Powder." Physical Review Letters. vol. 82, pp. 2278-81 (1991). https://doi.org/10.1103/PhysRevLett.82.2278.

[16] Fallert, J., Dietz, R. J. B., Hauser, M., and Stelzl, F. "Random lasing in ZnO nanocrystals.” Journal of Luminescence. vol. 129, pp. 1685-1688 (2009). http://dx.doi.org/10.1016/j.jlumin.2009.02.022.

[17] Ardakani, A., and Rafieipour, P. "Using $\mathrm{ZnO}$ nanosheets grown by electrodeposition in random lasers as scattering centers: the effects of sheet size and presence of mode competition." Journal of the Optical Society of America B. vol. 35, pp. 1708-1716 (2018). http://doi.org/10.1364/JOSAB.35.001708.

[18] Yang, H. Y., Lau, S. P., and Abiyasa, A. P. "High-temperature random lasing in ZnO nanoneedles.” Applied Physics Letters. vol. 89, pp. 011101-3 (2006). https://doi.org/10.1063/1.2219419.

[19] Weng, T. M., and Chang, T. H. et al. "Mode Control of random Laser Action Assisted by Whispering Gallery-Mode Resonance." ACS Photonics. vol. 1, pp. 12581263 (2014). https://pubs.acs.org/doi/full/10.1021/ph500201b\#.

[20] Nakamura, T., Sonoda, S., Yamamoto, T., and Adachi, S. "Discrete mode ZnO microparticle random laser.” Optics Letter. vol. 40, pp. 2661-2665 (2015). http://doi.org/10.1364/OL.40.002661.

[21] da Silva-Neto, M.L., de Oliveira, M.C.A., Dominguez, C.T. et al. "UV random laser emission from flexible $\mathrm{ZnO}-\mathrm{Ag}$-enriched electrospun cellulose acetate fiber matrix." Scientific Reports. vol. 9, 11765 (2019). https://doi.org/10.1038/s41598-019-48056w.

[22] Wang, C. S., Lin, H. Y., Lin, J. M., and Chen, Y. F. "Surface-Plasmon-Enhanced Ultraviolet Random Lasing from ZnO Nanowires Assisted by Pt Nanoparticles." Applied Physics Express. vol. 5, pp. 062003-3 (2012). https://iopscience.iop.org/article/10.1143/APEX.5.062003.

[23] Abiyasa, A. P., Yu, S. F., Lau, S. P., Leong, E., and Yang, H. Y. "Enhancement of ultraviolet lasing from Ag-coated highly disordered $\mathrm{ZnO}$ films by surface-plasmon resonance.” Applied Physics Letter. vol. 90, pp. 231106-3 (2007). https://doi.org/10.1063/1.2746940. 
[24] Firdaus, K., Nakamura, T., and Adachi, S. "Improved lasing characteristics of ZnO/organic dye random laser.” Applied Physics Letter. vol. 100, pp. 171101-4 (2012). http://doi.org/10.1063/1.4705471.

[25] Niyuki, R., Fujiwara, H., and Ishikawa, Y. "Torward single-mode random lasing within a submicrometre-sized spherical ZnO particle film." Journal of Optics. vol. 18 (2006). https://iopscience.iop.org/article/10.1088/2040-8978/18/3/035202.

[26] Fujiwara, H., Niyuki, R., Ishikawa, Y., Koshizaki, N., Tsuji, T. et al. "Low-threshold and quasi-single-mode random laser within a submicrometer-sized $\mathrm{ZnO}$ spherical particle film.” Applied Physics Letter. vol. 102, 061110 (2013). http://dx.doi.org/10.1063/1.4792349.

[27] Wu, X. H., Yamilov, A., Noh, H., and Cao, H. "Random lasing in closely packed resonant scatterers." Journal of the Optical Society of America B. vol. 21, pp. 159167 (2004). https://doi.org/10.1364/JOSAB.21.000159.

[28] “Zinc Oxide”. Wikipedia. (2021). http://en.wikipedia.org/wiki/Zinc oxide Wikipedia.

[29] "Silicon Oxide Nanoparticles/Nanopowder." American Elements: The Advanced Materials Manufacturer. (2018). https://www.americanelements.com/silicon-oxide nanoparticles-nanopowder-7631-86-9.

[30] "Polystyrene.” Wikipedia. 2018. https://en.wikipedia.org/wiki/Polystyrene.

[31] "Nitrogen Laser Operation Manual." Photon Technology International, Inc. (2008). www.pti-nj.com.

[32] "USB4000 Fiber Optics Spectrometer: Installation and Operation Manual." Ocean Optics Inc. 2006. http://focenter.com/wp-content/uploads/documents/Ocean-Optics Inc-Ocean Optics-USB4000-Fiber-Optic-Spectrometer-Fiber-Optic-Center.pdf.

[33] Peterson, Z., Word, R. C., and Könenkamp, R. "Structural instability and dynamic emission fluctuations in zinc oxide random lasers." Journal of Applied Physics. vol. 124 (2018). https://doi.org/10.1063/1.5037108.

[34] Subhash, N., Kartha, S. C., and Sathianandan, K. "New Vibrational Bands in Nitrogen Laser Emission Spectra.” Applied Optics. vol. 22, pp. 3612-3617 (1983). 